\title{
Galactic chemical evolution of sulphur
}

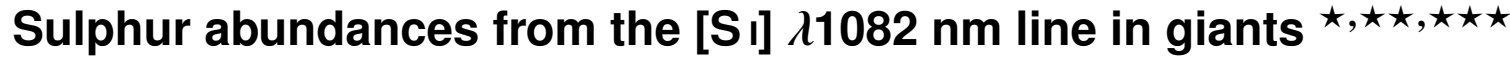

\author{
E. Matrozis ${ }^{1}$, N. Ryde ${ }^{1}$, and A. K. Dupree ${ }^{2}$ \\ ${ }^{1}$ Department of Astronomy and Theoretical Physics, Lund Observatory, Lund University, Box 43, 22100 Lund, Sweden \\ e-mail: ryde@astro.lu.se \\ 2 Harvard-Smithsonian Center for Astrophysics, 60 Garden St., Cambridge MA 02138, USA
}

Received 18 July 2013 / Accepted 29 August 2013

\begin{abstract}
Context. The Galactic chemical evolution of sulphur is still under debate. At low metallicities some studies find no correlation between $[\mathrm{S} / \mathrm{Fe}]$ and $[\mathrm{Fe} / \mathrm{H}]$, which is typical for $\alpha$-elements, while others find $[\mathrm{S} / \mathrm{Fe}]$ increasing towards lower metallicities, and still others find a combination of the two. Each scenario has different implications for the Galactic chemical evolution of sulphur.

Aims. The aim of this study is to contribute to the discussion on the Galactic chemical evolution of sulphur by deriving sulphur abundances from non-local thermodynamic equilibrium (LTE) insensitive spectral diagnostics in disk and halo stars with homogeneously determined stellar parameters.

Methods. We derived effective temperatures from photometric colours, surface gravities from stellar isochrones and Bayesian estimation, and metallicities and sulphur abundances from spectrum synthesis. We derived sulphur abundances from the $\left[\mathrm{S}_{\mathrm{I}}\right] \lambda 1082 \mathrm{~nm}$ line in 39 mostly cool and metal-poor giants using 1D LTE MARCS model atmospheres to model our high-resolution near-infrared spectra obtained with the VLT, NOT, and Gemini South telescopes.

Results. We derive homogeneous stellar parameters for 29 of the 39 stars. Our results argue for a chemical evolution of sulphur that is typical for $\alpha$-elements, contrary to some previous studies that have found high sulphur abundances $([\mathrm{S} / \mathrm{Fe}] \gtrsim 0.6)$ for stars with $-2.5<[\mathrm{Fe} / \mathrm{H}]<-1$. Our abundances are systematically higher by about 0.1 dex than those of other studies that arrived at similar conclusions using other sulphur diagnostics.

Conclusions. We find the $\left[\mathrm{S}_{\mathrm{I}}\right]$ line to be a valuable diagnostic of sulphur abundances in cool giants down to $[\mathrm{Fe} / \mathrm{H}] \simeq-2.3$. We argue that a homogeneous determination of stellar parameters is necessary, since the derived abundances are sensitive to them. Our results $([\mathrm{S} / \mathrm{Fe}])$ agree reasonably well with predictions of contemporary models of Galactic chemical evolution. In these models sulphur is predominantly created in massive stars by oxygen burning and is ejected into the interstellar medium during Type II supernovae explosions. Systematic differences with previous studies most likely fall within modelling uncertainties.
\end{abstract}

Key words. Galaxy: evolution - stars: fundamental parameters - stars: abundances - infrared: stars

\section{Introduction}

Sulphur is a chemical element of considerable scientific interest. First, it is one of the $\alpha$-elements, which are the elements from $\mathrm{O}$ to $\mathrm{Ti}$ with even atomic numbers. Studies of $\alpha$-element abundances in stellar atmospheres can shed light on important properties of stellar populations such as their star-formation history and initial mass function (e.g., McWilliam 1997). These properties are needed for the discussion on the formation and evolution of galaxies.

Second, sulphur is a volatile element. As such, it does not form dust easily, and the number of its atoms measured in a gas is indeed the total number, which makes sulphur suitable for cosmological studies. In particular, together with another volatile,

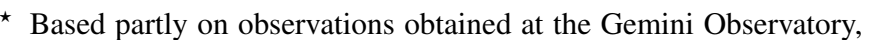
which is operated by the AURA, Inc., under a cooperative agreement with the NSF on behalf of the Gemini partnership: the NSF (US), the PPARC (UK), the NRC (Canada), CONICYT (Chile), the ARC, CNPq (Brazil), and CONICET (Argentina).

$\star \star$ Based partly on observations collected at the European Southern Observatory, Chile (ESO program 080.D-0675).

$\star \star \star$ Figures 3 and 4 are available in electronic form at http://www . aanda.org
}

zinc, it could be used as a cosmological clock for tracing the evolution of damped Ly $\alpha$ systems (Nissen et al. 2004, 2007) - huge clouds of predominantly neutral hydrogen gas at high redshifts that are thought to play an instrumental role in galaxy formation. Before this discussion can be engaged in, however, the chemical evolution of sulphur has to be understood in our own Galaxy.

Observational studies of Galactic chemical evolution of sulphur were scarce before the early 2000s due to the lack of suitable spectral diagnostics, and mainly concerned stars with [Fe/H] > -1 (e.g., Wallerstein \& Conti 1964; Francois 1987, 1988). This has changed partly because of the advent of infrared detector arrays. Now several studies on sulphur abundances in metal-poor stars have been published and left the matter of whether sulphur is a typical $\alpha$-element controversial (see Jönsson et al. 2011 for a review of the methods and findings of previous studies). To summarize, early studies by Israelian \& Rebolo (2001) and Takada-Hidai et al. (2002) found that the relative sulphur abundance continuously increases in the metallicity range from solar to at least $[\mathrm{Fe} / \mathrm{H}] \simeq-2.5$, implying a different chemical evolution history from that of other $\alpha$-elements, which display a (nearly) metallicity-independent enrichment with respect to iron in stars of low $([\mathrm{Fe} / \mathrm{H}] \lesssim-1)$ 
metallicity (Cayrel et al. 2004; Jonsell et al. 2005) ${ }^{1}$. A high rate of sulphur-rich hypernovae (e.g., Nakamura et al. 2001) and a time-delayed deposition of iron into the interstellar medium (Ramaty et al. 2000) have been proposed as possible explanations for these unexpected results. On the other hand, a number of studies (e.g, Ryde \& Lambert 2004; Korn \& Ryde 2005; Nissen et al. 2007; Spite et al. 2011) have found little to no evidence of high $([\mathrm{S} / \mathrm{Fe}] \gtrsim 0.6)$ sulphur abundances at any metallicity and instead argued that sulphur is a fairly typical $\alpha$-element and forms a plateau of $[\mathrm{S} / \mathrm{Fe}] \sim 0.3$ below $[\mathrm{Fe} / \mathrm{H}] \sim-1$, which points to an origin in Type II supernovae (e.g., Kobayashi et al. 2006). Finally, a combination of the two patterns, which implies a very complex history of chemical evolution of sulphur, has been found by Caffau et al. (2005, 2010).

It is open to debate whether there exist metal-poor stars with extremely high sulphur abundances, and the discrepant findings of previous studies could either reflect reality or differences in the employed analyses. Among these last are differences in sulphur diagnostics, stellar parameters and the types of stars, as well as assumptions regarding the dimensionality and local thermodynamic equilibrium (LTE; for a discussion on the effects of these assumptions see, e.g., Takada-Hidai et al. 2005; Takeda et al. 2005; Jönsson et al. 2011). In this 1D LTE study of sulphur abundances in cool giants we aim to mitigate some of these concerns.

1. Instead of adopting the stellar parameters $\left(T_{\mathrm{eff}}, \log g\right.$ and $[\mathrm{Fe} / \mathrm{H}])$ from multiple previous studies, as most of the studies of sulphur have done, we determined them using a single methodology to avoid introducing additional scatter in the results;

2. only the [S I] $\lambda 1082 \mathrm{~nm}$ line was used to derive sulphur abundances. This forbidden line is insensitive to the assumption of LTE, which sets at least one of the two main modelling assumptions - radial symmetry and local thermodynamic equilibrium - on a more solid footing.

\section{Observations}

While we are mainly interested in sulphur abundances in stars with $[\mathrm{Fe} / \mathrm{H}]<-1$, about half of our sample consists of more metal-rich stars. The sample is combined from four subsamples: 1) 14 stars with $[\mathrm{Fe} / \mathrm{H}]>-1$ from Ryde (2006) observed with the Gemini South telescope (Phoenix spectrometer) with $R=\lambda / \Delta \lambda \simeq 60000$ and $S / N \simeq 200 ; 2)$ the metal-poor $([\mathrm{Fe} / \mathrm{H}]<-1)$ sample observed by Ryde within the same programme, which was not published previously; 3) data from Jönsson et al. (2011) obtained at the VLT (CRIRES) with $R \simeq 80000$ and a signal-to-noise ratio $(\mathrm{S} / \mathrm{N})$ between about 250 and 500 for most stars (HD 13979 and HD 103545 have $\mathrm{S} / \mathrm{N}$ of 570 and 160 , respectively); 4) three metal-deficient $([\mathrm{Fe} / \mathrm{H}] \sim-0.4)$ stars observed by Ilyin (2000) with the NOT (SOFIN) with $R \simeq 80000$ and $S / N \simeq 70$. The wavelength coverage is about $5 \mathrm{~nm}(\lambda \simeq 1080-1085 \mathrm{~nm})$ for the Phoenix and SOFIN data and about $10 \mathrm{~nm}(\lambda \simeq 1080-1092 \mathrm{~nm}$ with a $2-\mathrm{nm}$ gap around $1086 \mathrm{~nm}$ ) for the CRIRES data. We refer to the publications of Ryde (2006), Jönsson et al. (2011) and Ilyin (2000) for detailed descriptions of subsamples 1-2, 3 and 4, respectively. Basic data of the sample stars are given in Table 1.

\footnotetext{
1 The abundance of an element $\mathrm{A}$ with respect to element $\mathrm{B}$ is $[\mathrm{A} / \mathrm{B}]=$ $\log _{10}\left(N_{\mathrm{A}} / N_{\mathrm{B}}\right)_{\star}-\log _{10}\left(N_{\mathrm{A}} / N_{\mathrm{B}}\right)_{\odot}$, where $N$ is the number density. We call $[\mathrm{Fe} / \mathrm{H}]$ the metallicity and $[\mathrm{S} / \mathrm{Fe}]$ the sulphur abundance.
}

\section{Analysis}

In this section we describe the determination of stellar parameters (Sect. 3.1) and sulphur abundances (Sect. 3.2).

\subsection{Stellar parameters}

\subsubsection{Effective temperature}

The effective temperatures were derived from the photometric colour-effective temperature calibrations of González Hernández \& Bonifacio (2009). In particular, we used photometric data from the Johnson and 2MASS photometric systems to derive $T_{\text {eff }}$ from the $B-V, V-J, V-H, V-K_{\mathrm{s}}$, and $J-K_{\mathrm{s}}$ colours.

Each colour gives an estimate of effective temperature $T_{\mathrm{eff}, i}=5040 \mathrm{~K} / \theta_{\mathrm{eff}, i}$, where

$\theta_{\mathrm{eff}, i}=a_{0}+a_{1} X_{i}+a_{2} X_{i}^{2}+a_{3} X_{i}[\mathrm{Fe} / \mathrm{H}]+a_{4}[\mathrm{Fe} / \mathrm{H}]+a_{5}[\mathrm{Fe} / \mathrm{H}]^{2}$,

$X_{i}$ is the (de-reddened) colour, and $a_{j}$ are the coefficients of the respective calibration. We then adopted a weighted mean effective temperature according to

$\bar{T}_{\mathrm{eff}}=\frac{\sum w_{i} T_{\mathrm{eff}, i}}{\sum w_{i}}$,

where $w_{i}=\left(\Delta T_{\mathrm{eff}, i}\right)^{-2}$. The uncertainties in the individual estimates $\left(\Delta T_{\mathrm{eff}, i}\right)$ are formally propagated uncertainties in the colours, metallicities, colour excesses, and the calibrations themselves. We adopted the weighted mean of the squared deviations from $\bar{T}_{\text {eff }}$ as the uncertainty of the temperature:

$\Delta \bar{T}_{\mathrm{eff}}=\frac{\sum w_{i}\left(T_{\mathrm{eff}, i}-\bar{T}_{\mathrm{eff}}\right)^{2}}{\sum w_{i}}$.

The Johnson $V$ magnitudes and $B-V$ colours and their uncertainties were taken from the General Catalogue of Photometric Data (GCPD; Mermilliod et al. 1997). The mean uncertainty in $V$ and $B-V$ for the sample is $\sim 0.014$ and $\sim 0.008$ mag, respectively, which motivated our assumption of $\Delta V=0.015$ and $\Delta(B-V)=0.010 \mathrm{mag}$ for stars that had no error estimates in the catalogue. A few stars (see Table 1) had no entries in GCPD, and their $V$ magnitudes and $B-V$ colours were taken from Rossi et al. (2005).

The 2MASS magnitudes and their uncertainties were taken from the 2MASS All-Sky Point Source Catalog (Skrutskie et al. 2006). The 2MASS magnitudes generally have good precision (quality flag A) for $J H K_{\mathrm{s}} \gtrsim 5$, which is the case for about half of our sample. For the other half the uncertainties are considerably larger, introducing an uncertainty of a few hundred $\mathrm{K}$ in each $T_{\mathrm{eff}, i}$. For these stars we chose to invert the colour transformations of Ramírez \& Meléndez (2005a) to derive the 2MASS $J H K_{\mathrm{s}}$ magnitudes from the $J H K$ magnitudes of the TCS photometric system (Alonso et al. 1994, 1998). The agreement between measured and calculated 2MASS magnitudes for faint stars was found to be excellent. While there is no good reason to assume that the transformation is valid for bright stars, we found good agreement between measured 2MASS magnitudes (with large uncertainties) and calculated-from-TCS 2MASS magnitudes (with small uncertainties), which indicates that the extrapolation is at the very least valid within the uncertainties of the observed magnitudes. Hence, in order of preference we adopted 1) measured 2 MASS magnitudes (when the quality flag was $A$ ); 2) TCS magnitudes transformed to the 2 MASS system (when the 
Table 1. Basic data of the analysed stars.

\begin{tabular}{|c|c|c|c|c|c|c|c|c|c|c|}
\hline \multicolumn{2}{|c|}{ Star identifier } & \multirow{2}{*}{$\begin{array}{l}\mathrm{RA}(\mathrm{J} 2000)^{a} \\
\quad(\mathrm{~h} \mathrm{~m} \mathrm{~s})\end{array}$} & \multirow{2}{*}{$\begin{array}{l}\operatorname{Dec}(\mathrm{J} 2000)^{a} \\
\quad(\mathrm{~d} \mathrm{~m} \mathrm{~s})\end{array}$} & \multirow{2}{*}{$\begin{array}{c}\pi^{a} \\
\text { (mas) }\end{array}$} & \multirow[t]{2}{*}{$V^{b}$} & \multirow[t]{2}{*}{$B-V^{b}$} & \multirow[t]{2}{*}{ Ref. $^{b}$} & \multirow[t]{2}{*}{$E_{B-V}^{c}$} & \multirow{2}{*}{$\begin{array}{l}J H K_{\mathrm{s}} \\
\text { flag }^{d}\end{array}$} & \multirow[t]{2}{*}{ Subsample } \\
\hline $\mathrm{HD} / \mathrm{BD}$ & HIP & & & & & & & & & \\
\hline 2796 & 2463 & 003116.915 & -164740.79 & $0.88 \pm 0.81$ & 8.495 & 0.747 & 1 & 0.020 & AAA & 2 \\
\hline 3546 & 3031 & 003833.345 & +291842.28 & $19.91 \pm 0.19$ & 4.361 & 0.871 & 1 & 0.008 & TCS & 1 \\
\hline 8724 & 6710 & 012617.594 & +170735.11 & $2.52 \pm 0.71$ & 8.300 & 0.987 & 1 & 0.106 & AAA & 2 \\
\hline 10380 & 7884 & 014125.894 & +052915.39 & $8.98 \pm 0.23$ & 4.437 & 1.364 & 1 & 0.016 & TCS & 1 \\
\hline 13979 & 10497 & 021520.853 & -255454.86 & $0.46 \pm 1.13$ & & & $\ldots$ & 0.012 & AAA & 3 \\
\hline 21581 & 16214 & 032854.486 & -002503.09 & $4.03 \pm 1.00$ & 8.710 & 0.825 & 1 & 0.069 & AAA & 3 \\
\hline 23798 & 17639 & 034645.722 & -305113.35 & $1.83 \pm 0.74$ & 8.302 & 1.098 & 1 & 0.008 & AAA & 3 \\
\hline 26297 & 19378 & 040903.418 & -155327.07 & $1.59 \pm 0.78$ & 7.470 & 1.110 & 1 & 0.030 & AEA & 3 \\
\hline 29574 & 21648 & 043855.733 & -132048.13 & $1.40 \pm 0.88$ & 8.336 & 1.401 & 1 & 0.183 & AEA & 3 \\
\hline 34334 & 24727 & 051810.569 & +332217.86 & $14.04 \pm 0.58$ & 4.538 & 1.266 & 1 & 0.014 & TCS & 4 \\
\hline 36702 & 25916 & 053152.230 & -383324.04 & $0.46 \pm 0.58$ & 8.365 & 1.216 & 1 & 0.028 & AAA & 3 \\
\hline 37160 & 26366 & 053654.389 & +091726.41 & $27.76 \pm 0.27$ & 4.082 & 0.958 & 1 & 0.008 & TCS & 4 \\
\hline 40460 & 28417 & 060006.039 & +271619.87 & $6.90 \pm 0.56$ & 6.609 & 1.022 & 1 & 0.026 & CCD & 1 \\
\hline 44007 & 29992 & 061848.528 & -145043.44 & $5.57 \pm 0.84$ & 8.059 & 0.839 & 1 & 0.033 & AAA & 3 \\
\hline 65953 & 39211 & 080113.336 & -012333.37 & $7.25 \pm 0.30$ & 4.676 & 1.491 & 1 & 0.009 & DDD & 4 \\
\hline 81192 & 46155 & 092445.336 & +194711.88 & $8.62 \pm 0.46$ & 6.530 & 0.947 & 1 & 0.016 & TCS & 1 \\
\hline 83212 & 47139 & 093619.952 & -205314.74 & $0.96 \pm 0.77$ & 8.335 & 1.070 & 1 & 0.059 & AAA & 3 \\
\hline 85773 & 48516 & 095339.242 & -225008.41 & $3.41 \pm 1.20$ & 9.380 & 1.120 & 1 & 0.027 & AAA & 3 \\
\hline 103545 & 58139 & 115527.163 & +090745.00 & $0.12 \pm 1.35$ & 9.200 & 0.710 & 2 & 0.034 & AAA & 3 \\
\hline 110184 & 61824 & 124014.079 & +083138.07 & $0.76 \pm 0.84$ & 8.305 & 1.175 & 1 & 0.020 & AAA & 2 \\
\hline 111721 & 62747 & 125125.196 & -132928.22 & $4.33 \pm 0.86$ & 7.971 & 0.799 & 1 & 0.038 & AAA & 2 \\
\hline 117876 & 66086 & 133248.214 & +242048.31 & $7.43 \pm 0.57$ & 6.092 & 0.965 & 1 & 0.008 & TCS & 1 \\
\hline 122563 & 68594 & 140231.846 & +094109.95 & $4.22 \pm 0.35$ & 6.200 & 0.904 & 1 & 0.019 & TCS & 2 \\
\hline 122956 & 68807 & 140513.026 & -145125.46 & $3.16 \pm 0.59$ & 7.220 & 1.010 & 1 & 0.065 & AEA & 2 \\
\hline 139195 & 76425 & 153629.579 & +100036.62 & $14.11 \pm 0.30$ & 5.260 & 0.945 & 1 & 0.011 & TCS & 1 \\
\hline 161074 & 86667 & 174228.362 & +243350.58 & $8.04 \pm 0.36$ & 5.525 & 1.452 & 1 & 0.028 & DCD & 1 \\
\hline 166161 & 88977 & 180940.687 & -084645.60 & $4.56 \pm 0.84$ & 8.120 & 0.976 & 1 & 0.293 & AAA & 2 \\
\hline 168723 & 89962 & 182118.601 & -025355.68 & $53.93 \pm 0.18$ & 3.254 & 0.941 & 1 & 0.016 & TCS & 1 \\
\hline 184406 & 96229 & 193405.353 & +072244.21 & $30.31 \pm 0.24$ & 4.450 & 1.177 & 1 & 0.005 & TCS & 1 \\
\hline 187111 & 97468 & 194839.575 & -120719.74 & $1.45 \pm 0.82$ & 7.720 & 1.225 & 1 & 0.124 & AEA & 2 \\
\hline 188512 & 98036 & 195518.792 & +062424.45 & $73.00 \pm 0.20$ & 3.715 & 0.855 & 1 & 0.003 & DCD & 1 \\
\hline 204543 & 106095 & 212928.213 & -033055.38 & $-0.13 \pm 1.08$ & 8.600 & 0.760 & 2 & 0.034 & AAA & 2 \\
\hline 212943 & 110882 & 222751.522 & +044144.41 & $21.99 \pm 0.37$ & 4.794 & 1.051 & 1 & 0.021 & TCS & 1 \\
\hline 214567 & 111810 & 223852.589 & +193120.16 & $8.74 \pm 0.44$ & 5.820 & 0.925 & 1 & 0.013 & DCD & 1 \\
\hline 216143 & 112796 & 225031.089 & -065449.54 & $0.87 \pm 0.91$ & 8.200 & 0.940 & 2 & 0.039 & AAA & 2 \\
\hline 219615 & 114971 & 231709.938 & +031656.23 & $23.64 \pm 0.18$ & 3.696 & 0.921 & 1 & 0.015 & DDD & 1 \\
\hline 220954 & 115830 & 232758.096 & +062244.37 & $21.96 \pm 0.25$ & 4.279 & 1.076 & 1 & 0.014 & TCS & 1 \\
\hline 221170 & 115949 & 232928.809 & +302557.86 & $2.94 \pm 0.69$ & 7.674 & 1.085 & 1 & 0.086 & AAA & 2 \\
\hline+302611 & 73960 & 150653.830 & +300036.95 & $1.07 \pm 1.23$ & 9.123 & 1.240 & 1 & 0.021 & AAA & 2 \\
\hline
\end{tabular}

Notes. ${ }^{(a)}$ Taken from van Leeuwen (2007); ${ }^{(b)}$ taken from 1 - Mermilliod et al. (1997), 2 - Rossi et al. (2005); (c) derived in this work (see Sect. 3.1.1); ${ }^{(d)}$ MASS photometry quality flags for colours $J, H$ and $K_{\mathrm{S}}$ (Skrutskie et al. 2006). TCS - colours transformed to the 2MASS system from the TCS system (see text). The $H$ magnitude of HD 26297, 29574 and 187111 was also transformed from the TCS system; ${ }^{(e)}$ as defined in Sect. 2 .

quality flag was $\mathrm{C}$ or poorer); 3) 2MASS magnitudes with quality flags of $\mathrm{C}$ or poorer when no TCS data were available.

The colours were de-reddened using the 3D Galactic extinction model of Drimmel et al. (2003). We used the IDL routine provided by the authors of the model to find $A_{V}$ along the line of sight to the position of the star, ${ }^{2}$ assumed $R_{V}=3.1$ (Cardelli et al. 1989) to calculate $E_{B-V}=A_{V} / R_{V}$, and adopted the extinction ratios $k=E_{X} / E_{B-V}$ from Ramírez \& Meléndez (2005b) to calculate the colour excess for the other colours.

The uncertainties of $E_{X}$ are large and difficult to estimate because $A_{V}, \pi, R_{V}$ and $k$ all have substantial uncertainties. Drimmel et al. (2003) stated that $\Delta A_{V}$ due to the model can be as large as $0.2 A_{V}$. We estimated the influence of the parallax uncertainties by calculating $A_{V}$ at the distance extremes allowed by the parallax uncertainties and found an additional relative uncertainty of $\Delta A_{V} / A_{V} \simeq 20 \%$ on average. Finally, while

\footnotetext{
$2 \mathrm{ftp}: / / \mathrm{ftp}$.to.astro.it/astrometria/extinction/
}

the assumption of a constant $R_{V}$ is not well justified, it is unlikely to deviate from the assumed value by more than $10 \%$ (McCall 2004). Taking these considerations into account, we assumed $\Delta E_{B-V}=0.3 E_{B-V}$ for all stars. The uncertainties of other colour excesses are slightly larger because of the $\sim 10 \%$ uncertainty in the extinction ratios (see, e.g., Taylor 1986).

\subsubsection{Surface gravity}

The surface gravity $\log g$ (cgs) can be computed from the fundamental relation

$\log g=\log g_{\odot}+\log \frac{M}{M_{\odot}}+\log \frac{L_{\odot}}{L}+4 \log \frac{T_{\text {eff }}}{T_{\text {eff } \odot \odot}}$

by deriving $T_{\text {eff }}$ and luminosity $L$ (from photometry and parallax), and then using theoretical stellar evolutionary tracks to obtain the mass $M$. Unfortunately, the parallax uncertainties are 
Table 2. Spectral lines of interest.

\begin{tabular}{|c|c|c|c|c|}
\hline \multirow{2}{*}{$\begin{array}{l}\lambda, \text { air } \\
(\mathrm{nm})\end{array}$} & \multirow[t]{2}{*}{ Transition } & \multirow{2}{*}{$\begin{array}{c}\chi \\
(\mathrm{eV})\end{array}$} & \multicolumn{2}{|c|}{$\log g f$} \\
\hline & & & (Ref.) & (adopted) \\
\hline \multicolumn{5}{|c|}{$\left[\mathrm{S}_{\mathrm{I}}\right]$} \\
\hline 1082.1176 & ${ }^{3} \mathrm{P}_{2}-{ }^{1} \mathrm{D}_{2}$ & 0.00 & -8.704 & -8.704 \\
\hline \multicolumn{5}{|c|}{$\mathrm{Fe}_{\mathrm{I}}$} \\
\hline 1081.8277 & ${ }^{3} \mathrm{D}_{1}^{\circ}-{ }^{3} \mathrm{P}_{1}$ & 3.96 & -1.948 & -2.212 \\
\hline 1083.3964 & ${ }^{5} \mathrm{D}_{3}-{ }^{3} \mathrm{~F}_{3}^{\circ}$ & 5.59 & -1.019 & -1.240 \\
\hline 1084.9462 & ${ }^{5} \mathrm{D}_{4}-{ }^{5} \mathrm{D}_{3}^{\circ}$ & 5.54 & -1.495 & -0.650 \\
\hline 1086.3519 & ${ }^{3} \mathrm{D}_{3}^{\circ}-{ }^{5} \mathrm{~F}_{4}$ & 4.73 & -0.903 & -1.000 \\
\hline 1088.1759 & ${ }^{3} \mathrm{P}_{1}{ }^{3}-{ }^{3} \mathrm{~F}_{2}^{\circ}$ & 2.85 & -3.553 & -3.383 \\
\hline 1088.4267 & ${ }^{3} \mathrm{D}_{2}^{\circ}-{ }^{3} \mathrm{P}_{2}$ & 3.93 & -1.927 & -2.075 \\
\hline 1089.6300 & ${ }^{3} \mathrm{P}_{1}-{ }^{3} \mathrm{P}_{2}^{\circ}$ & 3.07 & -2.692 & -2.822 \\
\hline \multicolumn{5}{|c|}{$\mathrm{Cr}_{\mathrm{I}}$} \\
\hline 1080.1362 & ${ }^{5} \mathrm{D}_{1}-{ }^{5} \mathrm{D}_{2}^{\circ}$ & 3.01 & -1.715 & -1.680 \\
\hline 1081.6901 & ${ }^{5} \mathrm{D}_{2}-{ }^{5} \mathrm{D}_{2}^{\circ}$ & 3.01 & -1.957 & -1.920 \\
\hline 1082.1658 & ${ }^{5} \mathrm{D}_{3}-{ }^{5} \mathrm{D}_{2}^{\circ}$ & 3.01 & -1.678 & -1.630 \\
\hline 1090.5712 & ${ }^{7} \mathrm{P}_{2}^{\circ}-{ }^{7} \mathrm{~S}_{3}{ }^{2}$ & 3.44 & -0.647 & -0.642 \\
\hline
\end{tabular}

Notes. Wavelengths, excitation energies, and oscillator strengths of the lines of interest. The line primarily used for metallicity determination is the Fe I $\lambda 1081.8 \mathrm{~nm}$ line. The reference is Froese Fischer \& Tachiev (2011) for the sulphur line and the Vienna Atomic Line Database (VALD; Kupka et al. 2000) for the others.

generally too large to effectively constrain $L$ and $M$ for the stars of this study (see Col. 5 of Table 1). Therefore, we used the PARAM tool ${ }^{3}$ (da Silva et al. 2006), which adopts two Bayesian priors to take into account the fact that the stars are not distributed randomly on the Hertzsprung-Russell diagram: 1) the masses are distributed according to the lognormal form of the Chabrier (2001) initial mass function; and 2) the star formation rate has been constant throughout the last 12 Gyr (the maximum age of a star). The theoretical stellar isochrones of Girardi et al. (2000) are then used to compute the probability density functions of $M$ and $\log g$, from which the mean surface gravity and its uncertainty are computed (see da Silva et al. 2006, for a detailed description of the method) and returned to the user. This probabilistic approach allows deriving precise surface gravities $(\Delta \log g \sim 0.1)$ even when the parallax errors are large $(\Delta \pi / \pi \sim 50 \%)$. However, we noticed that it breaks down when $\Delta \pi$ approaches and exceeds $\pi$, which is the case for seven stars of our sample.

\subsubsection{Metallicity}

Since the spectra analysed in this work cover a wavelength range of about $10 \mathrm{~nm}$ only, we were forced to derive the metallicity primarily from spectrum synthesis of a single $F_{I}$ line at $\lambda 1081.8 \mathrm{~nm}$. However, for about half of the stars we were able to use secondary metallicity diagnostics (given in Table 2) to verify that the metallicities are not in serious error.

Synthetic spectra were computed with the Spectroscopy Made Easy (SME; Valenti \& Piskunov 1996) software using the MARCS model atmospheres of Gustafsson et al. (2008). The main assumptions of the MARCS models are spherical symmetry, hydrostatic equilibrium, flux constancy, and local thermodynamic equilibrium. Convection is treated using the mixinglength formalism with mixing-length parameter $\alpha=\ell / H_{\mathrm{p}}=1.5$

http://stev.oapd.inaf.it/cgi-bin/param_1.1
( $H_{\mathrm{p}}$ is the local pressure scale height; Henyey et al. 1965). We used the $\alpha$-enhanced models for which $[\alpha / \mathrm{Fe}]$ linearly increases from 0 at solar metallicity and above to +0.4 at $[\mathrm{Fe} / \mathrm{H}]=-1$ and below. The abundances were derived by letting SME do multiple runs, in between which the desired abundances were adjusted until a satisfactory agreement between synthetic and observed spectra (determined by $\chi^{2}$ minimization) was achieved. The synthetic spectra were calculated by convolving the theoretical flux spectra with a Gaussian profile to account for macroturbulence and instrumental broadening. The abundances of other $\alpha$-elements in SME were fixed to be consistent with the MARCS models.

We derived astrophysical $\log g f$ values for the iron lines by fitting these lines in the solar centre intensity spectrum of Wallace et al. (1993, see Table 2). The resulting $\log g f$ values were tested by producing a synthetic flux spectrum of Arcturus for which the stellar parameters were taken from Ramírez \& Allende Prieto (2011). The observations (Hinkle et al. 1995) were well reproduced using the astrophysical $\log g f$ values - for the poorest-fitting line at $1088.2 \mathrm{~nm}$ the $\log g f$ value may be in error by about $0.05 \mathrm{dex}$, which, when assumed as the uncertainty in the $\log g f$ values, would increase the uncertainties in $[\mathrm{Fe} / \mathrm{H}]$ and $[\mathrm{S} / \mathrm{Fe}]$ by about another 0.02 dex. Astrophysical $\log g f$ values for four chromium lines in the region were also derived because the [ $\mathrm{S}_{\mathrm{I}}$ ] line is slightly blended by a $\mathrm{Cr}_{\mathrm{I}}$ line at $\lambda 1081.7 \mathrm{~nm}$ in the coolest/most metal-rich stars of our sample.

We included the molecular species $\mathrm{CN}$ (an up-to-date compilation privately provided by B. Plez), $\mathrm{C}_{2}$ (Querci et al. 1971; U. Jørgensen, priv. comm.), and CH (Jørgensen et al. 1996) in the computations of synthetic spectra.

In our methodology the metallicity is some function of $T_{\text {eff }}$ and $\log g$, which itself is some complicated function of $T_{\text {eff }}$. Due to this dependence the uncertainties cannot be added quadratically. But a conservative estimate of the uncertainties of the metallicities can be obtained by writing the full differential of $[\mathrm{Fe} / \mathrm{H}]\left(T_{\text {eff }}, \log g\right)$,

$\Delta[\mathrm{Fe} / \mathrm{H}]_{0}=\left|\frac{\partial[\mathrm{Fe} / \mathrm{H}]}{\partial T_{\text {eff }}} \Delta T_{\mathrm{eff}}\right|+\left|\frac{\partial[\mathrm{Fe} / \mathrm{H}]}{\partial \log g} \Delta \log g\right|$.

Here $\Delta T_{\text {eff }}$ and $\Delta \log g$ are given by Eq. (3) and PARAM, respectively. An additional uncertainty, mainly due to the uncertainty in continuum placement, is independently introduced by fitting the synthetic profile to the observed profile. This uncertainty was estimated to contribute $\Delta[\mathrm{Fe} / \mathrm{H}]_{\text {syn }}=0.03$ or 0.06 dex depending on the $\mathrm{S} / \mathrm{N}$ of the spectrum. The total uncertainty in metallicity was calculated as

$\Delta[\mathrm{Fe} / \mathrm{H}]=\sqrt{\left(\Delta[\mathrm{Fe} / \mathrm{H}]_{0}\right)^{2}+\left(\Delta[\mathrm{Fe} / \mathrm{H}]_{\mathrm{syn}}\right)^{2}}$.

The two partial derivatives in Eq. (5) were estimated by deriving the change in metallicity caused by changes in temperature (gravity) by $\delta T_{\text {eff }}= \pm 50 \mathrm{~K}$ and $\delta T_{\text {eff }}= \pm 100 \mathrm{~K}(\delta \log g=$ $\pm 0.2 \mathrm{dex}$ and $\delta \log g= \pm 0.4 \mathrm{dex}$ ). This gives four $\delta[\mathrm{Fe} / \mathrm{H}] / \delta T_{\text {eff }}$ $(\delta[\mathrm{Fe} / \mathrm{H}] / \delta \log g)$ values, one for each $50 \mathrm{~K}(0.2 \mathrm{dex})$ interval, and the mean of these four values was adopted as the approximation to $\partial[\mathrm{Fe} / \mathrm{H}] / \partial T_{\text {eff }}(\partial[\mathrm{Fe} / \mathrm{H}] / \partial \log g)$ at the given $T_{\text {eff }}(\log g)$.

Since $[\mathrm{Fe} / \mathrm{H}]$ has to be known before computing both $T_{\text {eff }}$ and $\log g$, it was first adopted from the literature (last column of Table 3; see Sect. 4). After the effective temperature and surface gravity were computed, the metallicity of the model atmosphere was adjusted until the best agreement between theoretical and observed spectra was obtained. Since this metallicity was almost 
Table 3. Stellar parameters derived in this and previous works.

\begin{tabular}{|c|c|c|c|c|c|c|c|c|c|c|c|c|}
\hline \multirow[t]{2}{*}{$\mathrm{HD} / \mathrm{BD}$} & \multicolumn{9}{|c|}{ This study } & \multicolumn{3}{|c|}{ Literature data } \\
\hline & $T_{\mathrm{eff}}$ & $\Delta T_{\mathrm{eff}}$ & $\log g$ & $\Delta \log g$ & {$[\mathrm{Fe} / \mathrm{H}]$} & $\Delta[\mathrm{Fe} / \mathrm{H}]$ & $i$ & $\xi$ & {$[(\mathrm{Fe}+\mathrm{Cr}) / \mathrm{H}]$} & $T_{\mathrm{eff}}$ & $\log g$ & {$[\mathrm{Fe} / \mathrm{H}]$} \\
\hline 2796 & 5002 & 30 & 1.73 & 0.20 & $(-2.33)$ & 0.13 & & 1.8 & & 4932 & 1.40 & -2.33 \\
\hline 3546 & 4977 & 30 & 2.44 & 0.12 & -0.54 & 0.04 & 1,2 & 1.4 & -0.64 & 4923 & 2.48 & -0.63 \\
\hline 8724 & 4790 & 30 & 1.66 & 0.12 & -1.55 & 0.07 & 1 & 1.8 & 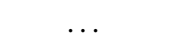 & 4574 & 1.35 & -1.75 \\
\hline 10380 & 4216 & 30 & 1.48 & 0.08 & -0.23 & 0.06 & 1 & 1.4 & -0.20 & 4121 & 1.72 & -0.27 \\
\hline 13979 & 5052 & 159 & $(1.50)$ & 0.49 & $(-2.57)$ & 0.24 & & 1.9 & $\ldots$ & 5043 & 1.50 & -2.57 \\
\hline 21581 & 5051 & 30 & 2.37 & 0.14 & -1.50 & 0.04 & $1,6,7$ & 1.6 & $\ldots$ & 4893 & 2.09 & -1.70 \\
\hline 23798 & 4482 & 33 & 0.71 & 0.12 & -2.10 & 0.07 & $1,5,6,7$ & 2.1 & $\ldots$ & 4442 & 0.79 & -2.12 \\
\hline 26297 & 4484 & 30 & 0.83 & 0.10 & -1.72 & 0.04 & $1,3,5,6$ & 2.0 & $\ldots$ & 4432 & 1.13 & -1.75 \\
\hline 29574 & 4299 & 30 & 0.51 & 0.34 & -1.97 & 0.06 & $1,3,5,6$ & 2.1 & & 4154 & 0.04 & -1.93 \\
\hline 34334 & 4308 & 30 & 1.87 & 0.06 & -0.40 & 0.05 & $1,3,4$ & 1.4 & -0.36 & 4194 & 2.17 & -0.37 \\
\hline 36702 & 4337 & 30 & $(0.88)$ & 0.10 & -2.12 & 0.07 & 1,3 & 2.0 & $\ldots$ & 4394 & 0.88 & -2.03 \\
\hline 37160 & 4771 & 30 & 2.57 & 0.05 & -0.59 & 0.06 & 1,3 & 1.3 & -0.56 & 4729 & 2.65 & -0.47 \\
\hline 40460 & 4724 & 45 & 2.42 & 0.08 & -0.21 & 0.05 & 1 & 1.2 & -0.29 & 4541 & 2.00 & -0.50 \\
\hline 44007 & 4907 & 30 & 2.36 & 0.07 & -1.67 & 0.06 & $1,3,6$ & 1.6 & $\ldots$ & 4862 & 2.10 & -1.64 \\
\hline 65953 & 4038 & 30 & 1.19 & 0.07 & -0.30 & 0.07 & 1 & 1.5 & -0.28 & 3960 & 1.68 & -0.36 \\
\hline 81192 & 4779 & 30 & 2.46 & 0.04 & -0.71 & 0.04 & 1,2 & 1.4 & -0.74 & 4728 & 2.58 & -0.64 \\
\hline 83212 & 4616 & 30 & 1.26 & 0.10 & -1.44 & 0.04 & $1,5,6$ & 1.8 & $\ldots$ & 4518 & 1.38 & -1.45 \\
\hline 85773 & 4443 & 30 & 1.00 & 0.12 & $(-2.24)$ & 0.24 & $\ldots$ & 2.0 & $\ldots$ & 4431 & 0.91 & -2.24 \\
\hline 103545 & 5085 & 60 & $(1.70)$ & 0.30 & -1.70 & 0.08 & 1 & 1.8 & $\ldots$ & 4708 & 1.70 & -2.14 \\
\hline 110184 & 4374 & 30 & $(0.65)$ & 0.40 & -2.33 & 0.10 & 1 & 2.1 & $\ldots$ & 4342 & 0.65 & -2.33 \\
\hline 111721 & 5078 & 30 & 2.56 & 0.17 & -1.30 & 0.07 & 1 & 1.5 & 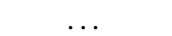 & 5038 & 2.70 & -1.34 \\
\hline 117876 & 4772 & 30 & 2.35 & 0.10 & -0.44 & 0.04 & 1,2 & 1.3 & -0.41 & 4722 & 2.27 & -0.49 \\
\hline 122563 & 4719 & 30 & 1.32 & 0.04 & $(-2.65)$ & 0.16 & $\ldots$ & 1.9 & $\ldots$ & 4594 & 1.25 & -2.65 \\
\hline 122956 & 4698 & 37 & 1.44 & 0.11 & -1.74 & 0.07 & 1 & 1.8 & $\ldots$ & 4618 & 1.50 & -1.74 \\
\hline 139195 & 4994 & 66 & 2.85 & 0.05 & 0.00 & 0.05 & 1,2 & 1.1 & -0.04 & 4991 & 3.03 & -0.11 \\
\hline 161074 & 3949 & 30 & 1.45 & 0.07 & -0.03 & 0.09 & 1 & 1.3 & -0.05 & 3997 & 1.83 & -0.27 \\
\hline 166161 & 5195 & 38 & 2.52 & 0.13 & -1.18 & 0.07 & 1 & 1.5 & & 5177 & 2.23 & -1.21 \\
\hline 168723 & 4936 & 30 & 3.04 & 0.03 & -0.22 & 0.04 & 1 & 1.1 & -0.21 & 4902 & 3.09 & -0.14 \\
\hline 184406 & 4530 & 30 & 2.70 & 0.06 & 0.20 & 0.04 & 1 & 1.0 & 0.18 & 4486 & 2.47 & -0.04 \\
\hline 187111 & 4418 & 30 & 0.70 & 0.11 & -1.78 & 0.04 & 1,3 & 2.1 & $\ldots$ & 4280 & 0.76 & -1.75 \\
\hline 188512 & 5111 & 79 & 3.52 & 0.06 & -0.23 & 0.06 & 1,2 & 1.2 & -0.21 & 5107 & 3.56 & -0.16 \\
\hline 204543 & 4490 & 30 & $(1.25)$ & 0.30 & -1.97 & 0.07 & 1 & 1.9 & $\ldots$ & 4682 & 1.25 & -1.82 \\
\hline 212943 & 4694 & 30 & 2.61 & 0.06 & -0.26 & 0.04 & 1,2 & 1.2 & -0.25 & 4611 & 2.79 & -0.29 \\
\hline 214567 & 4907 & 103 & 2.57 & 0.14 & -0.19 & 0.08 & 1,2 & 1.2 & -0.20 & 5003 & 2.50 & -0.03 \\
\hline 216143 & 4362 & 113 & (1.05) & 0.49 & -2.22 & 0.14 & 1 & 2.0 & $\ldots$ & 4511 & 1.05 & -2.22 \\
\hline 219615 & 4843 & 30 & 2.39 & 0.08 & -0.56 & 0.04 & 1 & 1.4 & -0.53 & 4848 & 2.52 & -0.49 \\
\hline 220954 & 4766 & 51 & 2.70 & 0.10 & 0.25 & 0.05 & 1 & 1.0 & 0.20 & 4708 & 2.74 & -0.03 \\
\hline 221170 & 4591 & 32 & 1.05 & 0.10 & -2.00 & 0.07 & 1 & 2.0 & $\ldots$ & 4480 & 0.98 & -2.11 \\
\hline+302611 & 4322 & 30 & $(0.90)$ & 0.33 & -1.43 & 0.07 & 1 & 1.9 & -1.41 & 4291 & 0.90 & -1.40 \\
\hline
\end{tabular}

Notes. The quantities in parentheses denote $\log g$ and $[\mathrm{Fe} / \mathrm{H}]$ values that were adopted from the literature (see the last two columns). $i$ denotes the $\mathrm{Fe}_{\mathrm{I}}$ lines used in metallicity determination (ordered according to wavelength as in Table 2). $\Delta \xi$ is about $0.2-0.3 \mathrm{~km} \mathrm{~s}^{-1}$. Literature data are the interquartile mean values taken from Soubiran et al. (2010).

always different from the one taken from the literature, the stellar parameters were then recalculated, starting with $T_{\text {eff }}$ and ending with $[\mathrm{Fe} / \mathrm{H}]$, until they converged to self-consistent values. One such iteration was typically sufficient to obtain convergence. This procedure led to an average reduction in the uncertainties of $T_{\text {eff }}$ and $\log g$ by about $10 \%$.

\subsubsection{Microturbulence}

There are too few iron lines to constrain the microturbulence from the spectra. However, since the lines of interest are weak, we were not concerned with precise values of $\xi$ and were content with estimating them from the $\xi=\xi\left(T_{\text {eff }}, \log g,[\mathrm{Fe} / \mathrm{H}]\right)$ calibrations privately provided by $\mathrm{M}$. Bergemann (derived for Gaia-ESO), which are accurate to about $0.2-0.3 \mathrm{~km} \mathrm{~s}^{-1}$ (standard deviation). A $0.3 \mathrm{~km} \mathrm{~s}^{-1}$ error in $\xi$ introduces a negligible error in the sulphur abundance $(0.02-0.03 \mathrm{dex})$ even for stars with the strongest sulphur lines (e.g., HD 65953).

\subsection{Sulphur abundances}

The sulphur abundances were derived from the $\left[\mathrm{S}_{\mathrm{I}}\right]$ line in the same way as the metallicities after all stellar parameters were determined. The atomic data of the sulphur line were adopted from the compilation of Froese Fischer \& Tachiev (2011).

The uncertainties in $[\mathrm{S} / \mathrm{Fe}]$ are

$\Delta[\mathrm{S} / \mathrm{Fe}]=\sqrt{\left(\Delta[\mathrm{S} / \mathrm{Fe}]_{0}\right)^{2}+\left(\Delta[\mathrm{S} / \mathrm{Fe}]_{\mathrm{syn}}\right)^{2}}$,

where $[\mathrm{S} / \mathrm{Fe}]_{0}$ is the uncertainty due to all stellar parameters (except for $\xi$ ) and is given by an expression analogous to Eq. (5) with a third term corresponding to $\Delta[\mathrm{Fe} / \mathrm{H}]$, and we assumed that $\Delta[\mathrm{S} / \mathrm{Fe}]_{\text {syn }}$ is also 0.03 or $0.06 \mathrm{dex}$ (because the iron and sulphur lines are of similar strength), but not necessarily the same as $\Delta[\mathrm{Fe} / \mathrm{H}]_{\text {syn. }}$. For most stars $\Delta[\mathrm{S} / \mathrm{Fe}]_{\text {syn }}$ is at least equal to $\Delta[\mathrm{Fe} / \mathrm{H}]_{\text {syn }}$ because generally it is more difficult to place the continuum for the sulphur line due to partial blending with 
the $\mathrm{Cr}$ I $\lambda 1082 \mathrm{~nm}$ line and molecular lines in the red and blue wings, respectively.

\section{Results}

The stellar parameters derived in this work are given in Table 3. For comparison, we also list the literature values, which are the interquartile mean (IQM) values of the entries in the PASTEL catalogue of stellar parameters (Soubiran et al. 2010) ${ }^{4}$.

All four stellar parameters were derived for 29 out of the 39 stars. For the remaining ten stars, all of which have $[\mathrm{Fe} / \mathrm{H}]<-1$, the IQM values of either the surface gravity or the metallicity (or both) were used. For seven of the ten stars the parallax errors exceed the measured parallax, and so we were unable to derive the surface gravity. We were unable to derive the metallicity for four of the ten stars because none of the iron lines were detected in their spectra.

Our sample covers effective temperatures from 3900 to $5200 \mathrm{~K}$, surface gravities mainly from 0.5 to 3.0 , and metallicities mainly from -2.4 to +0.2 . In general, the formally propagated temperature uncertainties are very small (between 30 and $50 \mathrm{~K}$ ), which indicates that the individual colours give consistent estimates of $T_{\text {eff. }}$ Larger uncertainties are associated with fewer colours used or might indicate problems with reddening corrections. The lower limit of $30 \mathrm{~K}$ is artificial - for most stars Eq. (3) gives a smaller uncertainty of the effective temperature, which can render the derived surface gravities very sensitive to the exact value of this uncertainty. Therefore, $\Delta T_{\text {eff }}$ was adjusted for every star to equal the result of Eq. (3) or $30 \mathrm{~K}$, whichever was larger, before we calculated its surface gravity, metallicity, and sulphur abundance. $\Delta \log g$ are typically $0.1-0.2$ dex. Higher values $(\sim 0.4 \mathrm{dex})$ are characteristic for the seven stars with IQM gravities (their uncertainties were calculated as the interquartile range (IQR) divided by 1.35 , which would equal $1-\sigma$ uncertainties for normally distributed values). Uncertainties in $[\mathrm{Fe} / \mathrm{H}]$ are normally below $0.1 \mathrm{dex}$, but again higher values are typical for the four stars with IQM metallicities.

In abundance studies of low-mass main-sequence stars a very tight correlation between the chromium and iron abundances is observed (Bensby et al. 2005), with $[\mathrm{Cr} / \mathrm{H}] \simeq[\mathrm{Fe} / \mathrm{H}]$ in stars with $[\mathrm{Fe} / \mathrm{H}]>-1$. Under the reasonable assumption that this correlation extends to giants, we calculated updated metallicities by treating the chromium abundance as another measure of the metallicity ${ }^{5}$. The updated values are given in Col. 9 of Table 3 . As shown by the very small changes in the metallicity, the $\mathrm{Cr}$ and $\mathrm{Fe}$ abundances are indeed nearly equal for the most part. However, to remain consistent throughout the metallicity range, the updated metallicities are not considered further.

The stellar parameters derived in this study are plotted against their respective IQM values in Fig. 1. Open circles are used for stars with homogeneous stellar parameters, crosses for stars with IQM surface gravities, and triangles for stars with IQM metallicities. The jagged dashed line denotes the uncertainty in the literature values, which is equal to the IQR value divided by 1.35 . The error bars are calculated from Eq. (3) for $T_{\text {eff }}$ (top panel; note that some are clearly smaller than the minimum

\footnotetext{
4 Whenever a single value of one of the stellar parameters was referenced multiple times, all but one of the duplicate values from different studies by the same first author were discarded to reduce the influence of a single study.

5 Logarithm of the number of chromium atoms per $10^{12}$ hydrogen atoms in the Sun: $\log \epsilon(\mathrm{Cr})_{\odot}=5.64$ (Grevesse et al. 2007).
}
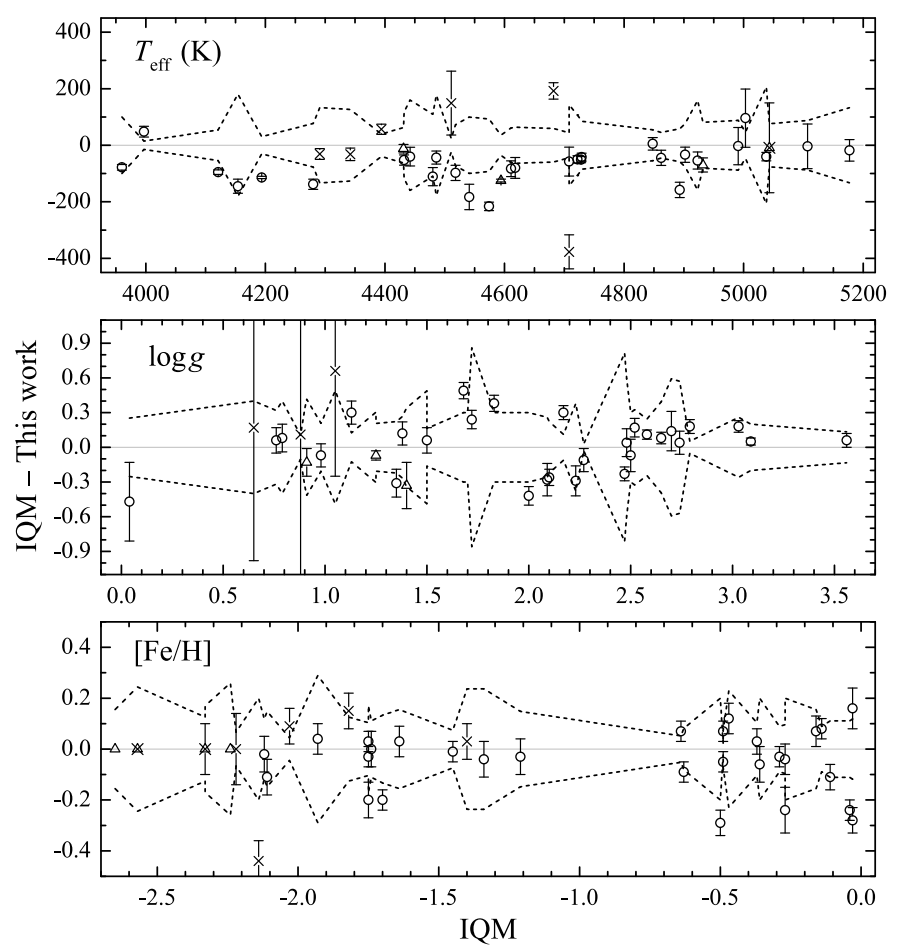

Fig. 1. Differences between stellar parameters from the literature (interquartile mean (IQM) values taken from Soubiran et al. 2010) and the stellar parameters derived in this study versus the literature parameters - top: effective temperatures; middle: surface gravities; bottom: metallicities. The circles correspond to stars with homogeneous stellar parameters, crosses (triangles) denote stars whose gravities (metallicities) were adopted from the literature. The error bars are given by Eq. (3), PARAM (see text) and Eq. (6) for the three respective stellar parameters. The jagged line indicates the uncertainty of the literature values (IQR/1.35).

adopted $\Delta T_{\text {eff }}$ of $30 \mathrm{~K}$ ), returned by PARAM for $\log g$ (middle panel), and calculated from Eq. (6) for $[\mathrm{Fe} / \mathrm{H}]$ (bottom panel).

The sulphur abundances derived in this work are listed in the third column of Table 4 and are plotted versus metallicities in Fig. 2. The symbols are the same as in Fig. 1. The starting points of the horizontal (vertical) arrows indicate the upper limits of the metallicities (sulphur abundances). For reference, the abundance of the other $\alpha$-elements adopted for spectrum synthesis is denoted by the solid line. The lack of stars with $-1.2<$ $[\mathrm{Fe} / \mathrm{H}]<-0.8$ is a chance outcome. While typical uncertainties of $[\mathrm{S} / \mathrm{Fe}]$ are $0.1-0.15 \mathrm{dex}, \Delta[\mathrm{S} / \mathrm{Fe}]$ can easily reach $\sim 0.4 \mathrm{dex}$ when the stellar parameters are poorly constrained. Some examples of the theoretical spectra (red lines) fitted to observations (black circles) are presented in Figs. 3 and 4, in which a 1-nm wide spectral region containing the forbidden sulphur line is shown. This region also contains the $\lambda 1081.8 \mathrm{~nm}$ iron line used to derive metallicities and two of the four chromium lines listed in Table 2. The positions of these spectral lines are indicated by vertical dashed lines.

\section{Discussion}

In this section we discuss some points concerning our stellar parameters (Sect. 5.1) and sulphur abundances (Sect. 5.2), compare our results with previous studies on the Galactic chemical evolution of sulphur (Sect. 5.2.1), and end by comparing our results with theoretical expectations (Sect. 5.2.2). 
E. Matrozis et al.: Galactic chemical evolution of sulphur

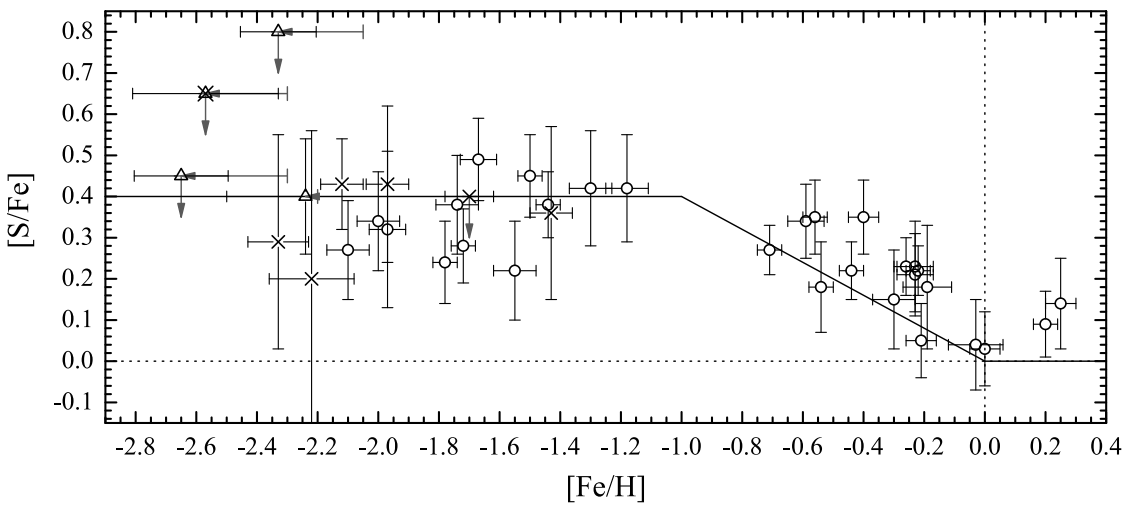

Fig. 2. Sulphur abundances derived in this work. The symbols are the same as in Fig. 1. The starting points of the horizontal (vertical) arrows indicate the upper limit of the metallicity (sulphur abundance). The (upper limit of) sulphur abundance for these stars is derived with respect to the literature (IQM) metallicity. The solid line shows the enrichment assumed for the other $\alpha$-elements. The dotted lines cross at the solar values, which correspond to $\log \epsilon(\mathrm{Fe})_{\odot}=7.45$ and $\log \epsilon(\mathrm{S})_{\odot}=7.14$ (Grevesse et al. 2007).
Table 4. Sulphur abundances derived in this and previous works.

\begin{tabular}{|c|c|c|c|c|c|}
\hline \multirow[t]{2}{*}{$\mathrm{HD} / \mathrm{BD}$} & \multicolumn{3}{|c|}{ This study } & \multicolumn{2}{|c|}{ Previous studies } \\
\hline & {$[\mathrm{Fe} / \mathrm{H}]$} & {$[\mathrm{S} / \mathrm{Fe}]$} & $\Delta[\mathrm{S} / \mathrm{Fe}]$ & {$[\mathrm{Fe} / \mathrm{H}]$} & {$[\mathrm{S} / \mathrm{Fe}]$} \\
\hline 8724 & -1.55 & 0.22 & 0.12 & $\cdots$ & $\cdots$ \\
\hline 34334 & -0.40 & 0.35 & 0.09 & $\ldots$ & $\ldots$ \\
\hline 37160 & -0.59 & 0.34 & 0.09 & $\ldots$ & $\ldots$ \\
\hline 65953 & -0.30 & 0.15 & 0.12 & $\ldots$ & $\ldots$ \\
\hline 110184 & -2.33 & 0.29 & 0.26 & $\ldots$ & $\ldots$ \\
\hline 122956 & -1.74 & 0.38 & 0.12 & $\ldots$ & $\ldots$ \\
\hline 166161 & -1.18 & 0.42 & 0.13 & $\ldots$ & $\ldots$ \\
\hline 204543 & -1.97 & 0.43 & 0.19 & $\ldots$ & $\ldots$ \\
\hline+302611 & -1.43 & 0.36 & 0.21 & $\ldots$ & $\ldots$ \\
\hline \multicolumn{6}{|c|}{ Ryde \& Lambert (2004) } \\
\hline 111721 & -1.30 & 0.42 & 0.14 & -1.21 & 0.32 \\
\hline \multicolumn{6}{|c|}{ Takada-Hidai et al. (2005) } \\
\hline 187111 & -1.78 & 0.24 & 0.10 & -1.85 & 0.66 \\
\hline 216143 & -2.22 & 0.20 & 0.36 & -2.15 & 0.37 \\
\hline 221170 & -2.00 & 0.34 & 0.12 & -2.10 & 0.47 \\
\hline \multicolumn{6}{|c|}{ Ryde (2006) } \\
\hline 3546 & -0.54 & 0.18 & 0.11 & -0.60 & 0.25 \\
\hline 10380 & -0.23 & 0.21 & 0.10 & -0.19 & 0.03 \\
\hline 40460 & -0.21 & 0.05 & 0.09 & -0.44 & 0.00 \\
\hline 81192 & -0.71 & 0.27 & 0.06 & -0.56 & 0.15 \\
\hline 117876 & -0.44 & 0.22 & 0.07 & -0.44 & 0.10 \\
\hline 139195 & 0.00 & 0.03 & 0.09 & 0.02 & -0.14 \\
\hline 161074 & -0.03 & 0.04 & 0.11 & -0.21 & 0.20 \\
\hline 168723 & -0.22 & 0.22 & 0.06 & -0.13 & 0.14 \\
\hline 184406 & 0.20 & 0.09 & 0.08 & 0.07 & -0.06 \\
\hline 188512 & -0.23 & 0.23 & 0.11 & -0.11 & 0.20 \\
\hline 212943 & -0.26 & 0.23 & 0.07 & -0.28 & 0.21 \\
\hline 214567 & -0.19 & 0.18 & 0.15 & 0.09 & -0.08 \\
\hline 219615 & -0.56 & 0.35 & 0.09 & -0.36 & 0.24 \\
\hline 220954 & 0.25 & 0.14 & 0.11 & -0.04 & 0.09 \\
\hline \multicolumn{6}{|c|}{ Spite et al. (2011) } \\
\hline 2796 & $(-2.33)$ & $\lesssim 0.80$ & - & -2.41 & 0.31 \\
\hline 122563 & $(-2.65)$ & $\lesssim 0.45$ & - & -2.76 & 0.38 \\
\hline \multicolumn{6}{|c|}{ Jönsson et al. (2011) } \\
\hline 13979 & $(-2.57)$ & $\lesssim 0.65$ & - & -2.26 & $\lesssim 0.21$ \\
\hline 21581 & -1.50 & 0.45 & 0.10 & -1.64 & 0.52 \\
\hline 23798 & -2.10 & 0.27 & 0.12 & -2.03 & 0.37 \\
\hline 26297 & -1.72 & 0.28 & 0.09 & -1.51 & 0.41 \\
\hline 29574 & -1.97 & 0.32 & 0.19 & -1.70 & 0.27 \\
\hline 36702 & -2.12 & 0.43 & 0.11 & -2.06 & 0.45 \\
\hline 44007 & -1.67 & 0.49 & 0.10 & -1.65 & 0.37 \\
\hline 83212 & -1.44 & 0.38 & 0.08 & -1.40 & 0.41 \\
\hline 85773 & $(-2.24)$ & 0.40 & 0.14 & -2.36 & 0.53 \\
\hline 103545 & -1.70 & $\lesssim 0.40$ & - & -2.14 & $\lesssim 0.52$ \\
\hline
\end{tabular}

Notes. The quantities in parentheses denote values that were adopted from the literature (last column of Table 3). The metallicities and abundances of previous studies have been rescaled to correspond to $\log \epsilon(\mathrm{Fe})_{\odot}=7.45$ and $\log \epsilon(\mathrm{S})_{\odot}=7.14$ (Grevesse et al. 2007).

\subsection{Stellar parameters}

We found no $T_{\text {eff }}$-dependent differences between the effective temperatures derived from the González Hernández \& Bonifacio (2009) calibrations and the interquartile mean effective temperatures. However, the former are systematically hotter by $65 \pm$ $66 \mathrm{~K}$ (standard deviation). We also derived the effective temperatures from the calibrations of Ramírez \& Meléndez (2005b) and found no statistically significant differences from the IQM temperatures on average. We opted to use the González Hernández \& Bonifacio (2009) calibrations for subsequent work because the metallicity binning for each colour used by Ramírez \& Meléndez (2005b) results in the calibrated temperatures being discontinuous across the edges of the bins, and a change as small as $0.01 \mathrm{dex}$ in $[\mathrm{Fe} / \mathrm{H}]$ can change $T_{\text {eff }}$ by $\sim 30 \mathrm{~K}$ for some colours.

The surface gravities and metallicities are found to very closely agree on average with the IQM gravities and metallicities (to within 0.05 dex).

No non-LTE corrections were applied to the metallicities derived in this work because we are not aware of any non-LTE calculations of $\mathrm{Fe}_{\mathrm{I}}$ lines with wavelengths $\lambda>1000 \mathrm{~nm}$.

\subsection{Sulphur abundances}

We plot the sulphur abundances versus metallicities of the whole sample in Fig. 2. In contrast to previous studies by Israelian \& Rebolo (2001), Takada-Hidai et al. (2002), Caffau et al. (2005, 2010), no stars with extremely high sulphur abundances are observed, nor are the sulphur abundances found to increase towards lower metallicities. In fact, the opposite is observed - a linear fit for the metal-poor $([\mathrm{Fe} / \mathrm{H}]<-1)$ stars with homogeneous stellar parameters gives $[\mathrm{S} / \mathrm{Fe}]=0.17[\mathrm{Fe} / \mathrm{H}]+0.63$ - although the significance is low (the standard error of the slope is 0.15) and, when the stars with IQM gravities are included, the trend becomes shallower and insignificant (the slope becomes $0.07 \pm 0.12$ ). If the slope is fixed at 0 , we derive an average abundance of $[\mathrm{S} / \mathrm{Fe}]=0.35 \pm 0.09$ (standard deviation) for $-2.4<[\mathrm{Fe} / \mathrm{H}]<-1.1$, which is slightly below the $[\alpha / \mathrm{Fe}]=0.4$ of the MARCS models. For $-0.8<[\mathrm{Fe} / \mathrm{H}]<$ $0.0[\mathrm{~S} / \mathrm{Fe}]$ is found to decrease as the metallicity increases, which is expected of $\alpha$-elements (solid line). But note that in reality more complicated patterns are observed than are assumed in the $\alpha$-enhanced MARCS models - above $[\mathrm{Fe} / \mathrm{H}] \simeq-0.8$ two distinct trends, corresponding to the thin and thick disks, are observed (Bensby et al. 2005; Fuhrmann 2008). According to the models of Kobayashi et al. (2011), the differences for the sulphur trends are less than 0.05 dex, however.

The uncertainty introduced in the final results $([\mathrm{S} / \mathrm{Fe}])$ is likely to be large when IQM values of stellar parameters are 


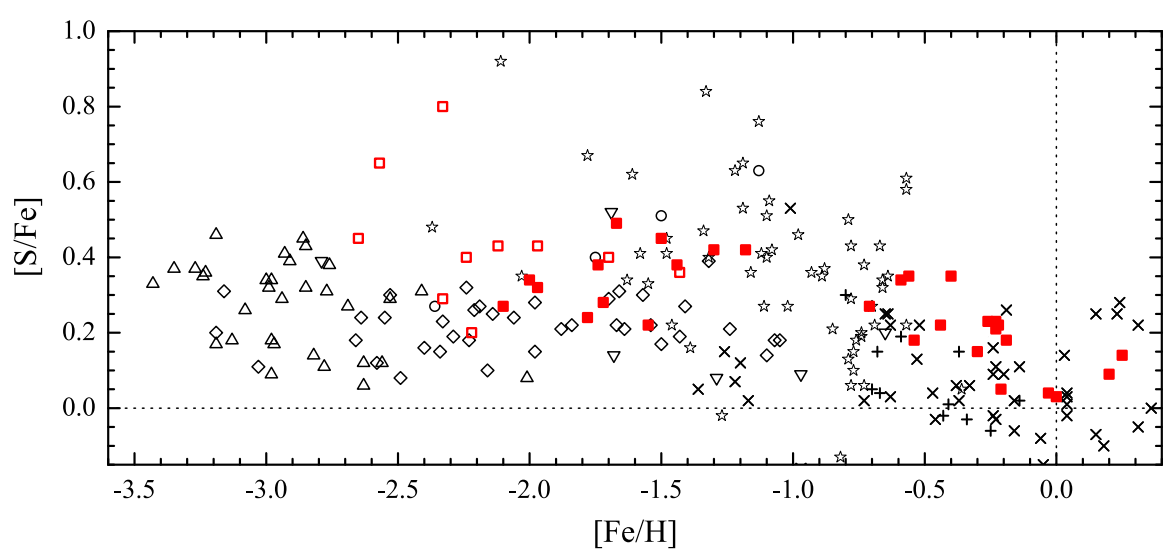

Fig. 5. Sulphur abundances derived in this and previous works. Our results are plotted as red squares (solid for stars with homogeneously determined stellar parameters). The symbols denote measurements from Chen et al. (2002, plus symbols), Takada-Hidai et al. (2002, crosses), Ryde \& Lambert (2004, downward-pointing triangles), Caffau et al. (2005, stars), Nissen et al. (2007, rhombi), Caffau et al. (2010, circles), and Spite et al. (2011, upward-pointing triangles). used. However, the IQM values might still be preferred to adopting the values from individual studies unless the missing parameters can be adopted from a single study.

Our formally propagated uncertainties of $[\mathrm{S} / \mathrm{Fe}]$ are similar to what is usually simply stated in the literature: $\Delta[\mathrm{S} / \mathrm{Fe}] \sim$ $0.10-0.15$ dex. Nevertheless, a detailed breakdown of individual contributions has allowed us to establish that $\log g$ and $[\mathrm{Fe} / \mathrm{H}]$ are the largest contributors to the uncertainty of $[\mathrm{S} / \mathrm{Fe}]$, each introducing a partial error of about $0.05-0.10$ dex for most stars. While the forbidden line exhibits a weak temperature sensitivity (the partial error caused by $\Delta T_{\text {eff }}$ is only about $0.02 \mathrm{dex}$ ), our methodology does not take full advantage of this fact, because we used the temperature-sensitive $\mathrm{Fe}_{\mathrm{I}}$ lines to derive the metallicity. Using lines of singly-ionized iron instead would address this flaw and also reduce the possibility that deviations from LTE affect the results (Mashonkina et al. 2011; Lind et al. 2012). Unfortunately, no suitable Fe II lines are located in the spectral region around the sulphur line. Overall, we found that the uncertainties in stellar parameters usually dominate the total uncertainty of $[\mathrm{S} / \mathrm{Fe}]$.

Obviously, deriving abundances from a single sulphur line renders each individual of our results more vulnerable to errors, especially at the lowest $([\mathrm{Fe} / \mathrm{H}] \lesssim-2)$ metallicities where the risk of mistaking noise for the iron or sulphur line is increased, and at the highest metallicities because of the possibility of unaccounted weak blends. As stated before, to avoid severe errors in our metallicity estimates, we checked whether the metallicities derived from the $\mathrm{Fe}_{\mathrm{I}} \lambda 1081.8 \mathrm{~nm}$ line are consistent with other, less prominent iron lines in the region whenever possible.

\subsubsection{Comparison with previous studies}

In addition to the Ryde (2006) and Jönsson et al. (2011) samples, we have some stars in common with Ryde \& Lambert (2004), Takada-Hidai et al. (2005), and Spite et al. (2011), all of whom derived the sulphur abundances from the $S_{\text {I }} \lambda 923 \mathrm{~nm}$ lines - Takada-Hidai et al. (2005) used equivalent widths, and Ryde \& Lambert (2004) and Spite et al. (2011) performed spectrum synthesis. We list our results along with these previous results in Table 4. The metallicities and sulphur abundances of previous studies have been translated to the solar chemical composition scale of Grevesse et al. (2007): $\log \epsilon(\mathrm{Fe})_{\odot}=7.45$ and $\log \epsilon(\mathrm{S})_{\odot}=7.14$. This scale was adopted to be consistent with the MARCS models. Adopting the solar abundances from the more recent study of Asplund et al. (2009) would shift all metallicities and sulphur abundances by -0.05 dex and +0.07 dex, respectively.

Clearly, there can be major differences in the metallicities and sulphur abundances for individual stars analysed in separate studies. We tested to what extent these differences can be explained by the differences in the stellar parameters. This is particularly important in cases of Ryde (2006) and Jönsson et al. (2011), because these studies analysed the same observational data and used the same spectral diagnostics as we did. And indeed, we find that the differences in $[\mathrm{S} / \mathrm{Fe}]$ can be explained to within $\sim 0.05$ dex after accounting for the differences in stellar parameters. In other words, we can reproduce their results when we adopt the same stellar parameters. The answer to the question of which sulphur abundances are the "correct ones" therefore depends on which stellar parameters one trusts more. Given the significant differences from study to study, the necessity for homogeneous stellar parameters is apparent. Nevertheless, note that systematic differences between stellar parameters from multiple literature sources may not have a noticeable effect on the main conclusions. For example, Jönsson et al. (2011) found a plateau in $[\mathrm{S} / \mathrm{Fe}]$ with a scatter (standard deviation) of $0.085 \mathrm{dex}$. We obtain virtually the same scatter for their stars (0.080 dex).

Accounting for the differences in stellar parameters is not always enough when comparing the results with the other studies. For example, consider HD 187111, for which we derive a lower [S/Fe] (by 0.42 dex) than Takada-Hidai et al. (2005) when adopting a higher $T_{\text {eff }}$ (by $100 \mathrm{~K}$ ), lower gravity (by $0.4 \mathrm{dex}$ ), and slightly higher metallicity (by $0.07 \mathrm{dex}$ ). Differences in stellar parameters account for about a half of the discrepancy in [S/Fe], leaving a difference of about 0.2 dex. This remaining difference can be well explained by the strong non-LTE effects of the triplet - the calculations by Takeda et al. (2005) indicate that the result of Takada-Hidai et al. (2005) should be lowered by 0.15 dex. Thus, we argue that HD 187111 has a much lower sulphur abundance than reported in Takada-Hidai et al. (2005).

In some other cases the differences cannot be explained so easily, and remaining differences, which can be about 0.2 dex in [S/Fe], must be caused by a combination of a number of factors, for example, the 1) quality of observations; 2) accuracy of continuum normalization; 3) completeness and accuracy of atomic and molecular data; 4) uncertainty in the non-LTE corrections; 5) differences in 3D effects; 6) different software used for computing synthetic spectra; or 7) different versions of the model atmospheres.

Our mean sulphur abundance below $[\mathrm{Fe} / \mathrm{H}]=-1([\mathrm{~S} / \mathrm{Fe}] \simeq$ $0.35)$ is significantly $(\sim 2-3 \sigma)$ higher than found in previous studies by Nissen et al. (2007) and Spite et al. (2011), who found mean $[\mathrm{S} / \mathrm{Fe}] \simeq 0.22$ and 0.27 dex, respectively (see Fig. 5). There are three key differences between the studies: 1) the methods used to obtain the stellar parameters in the three studies are different. Nissen et al. (2007) derived $T_{\text {eff }}$ from the $\mathrm{H} \beta$ line, $\log g$ from Eq. (4) and $[\mathrm{Fe} / \mathrm{H}]$ from equivalent widths of Fe II lines. 


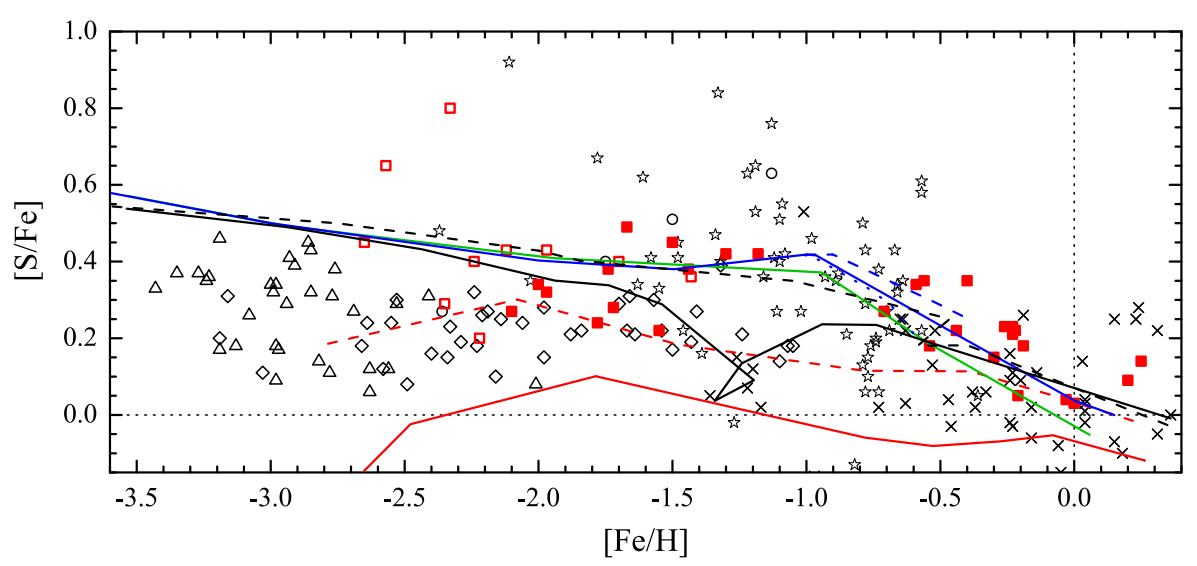

Fig. 6. Comparison with theoretical models of Galactic chemical evolution. The symbols corresponding to previous studies are the same as in Fig. 5. Our results are plotted with solid and empty red squares. The red solid and dashed lines correspond to the Timmes et al. (1995) nominal model and one where the iron yields are reduced by a factor of two, respectively. The green line is the prediction of Kobayashi et al. (2006) for the solar neighbourhood. The dotted, solid and dashed blue lines correspond to halo, solar neighbourhood, and thick disk models of Kobayashi et al. (2011), respectively. The solid and dashed black lines correspond to the Brusadin et al. (2013) double-infall model with and without outflow, respectively.
Spite et al. (2011) adopted stellar parameters primarily from three different studies, in which $T_{\text {eff }}$ was derived from photometric colours and $\log g$ from ionization equilibrium. 2) We used the forbidden line in our analysis, while Nissen et al. (2007) and Spite et al. (2011) used the $\mathrm{S}_{\text {I }}$ triplet at $923 \mathrm{~nm}$. 3) Our sample and that of Spite et al. (2011) consist mainly of giants and subgiants, while the sample of Nissen et al. (2007) mainly comprises dwarf stars. A systematic difference between giants and dwarfs would be a puzzle to solve for model atmospheres. While such a difference may exist, the sulphur abundances derived by Spite et al. (2011) for the few dwarf stars in their sample agree well with their results for giants, which argues against this possibility. Systematic differences between the stellar parameter scales and sulphur diagnostics, most likely in the form of non-LTE and 3D corrections, are more probable. Calculations by Takeda et al. (2005) and Korotin (2009) have shown that the non-LTE corrections are extremely dependent on temperature and metallicity for metal-poor stars (e.g., the non-LTE corrections for stars with $T_{\text {eff }}=5000$ and $5500 \mathrm{~K}$ at $[\mathrm{Fe} / \mathrm{H}]=-2$ are $\Delta[\mathrm{S} / \mathrm{Fe}] \simeq-0.4$ and $\Delta[\mathrm{S} / \mathrm{Fe}] \simeq-0.6$ dex, respectively, and at $[\mathrm{Fe} / \mathrm{H}]=-3$ they are $\Delta[\mathrm{S} / \mathrm{Fe}] \simeq-0.4$ and $\Delta[\mathrm{S} / \mathrm{Fe}] \simeq-0.8 \mathrm{dex}$, respectively). It is possible that the non-LTE corrections for the triplet are systematically too large by about 0.1 dex for these types of stars. On a suggestive side note, Jönsson et al. (2011) found their non-LTEcorrected sulphur abundances from the triplet at $\lambda 1045 \mathrm{~nm}$ to be lower by an average of 0.06 dex (compared with the abundances derived from the forbidden line) in stars of subsample 3. On the other hand, while we emphasize again that the $\left[\mathrm{S}_{\mathrm{I}}\right]$ line forms in LTE, deviations from LTE are probably still important for our sulphur abundances because of their effects on the Fe I lines that were used to derive the metallicities. As stated before, the non-LTE effects of the iron lines with $\lambda>1000 \mathrm{~nm}$ have not been studied.

We also recall that no attempts have been made to account for 3D effects on the results of this study. Jönsson et al. (2011) used the 3D hydrodynamic model atmospheres of Collet et al. (2007) to estimate that a typical 3D correction for subsample 3 stars might lower the sulphur abundances by about $0.05-0.15$ dex, which brings our results in better agreement with previous studies. Negative corrections for the $\left[\mathrm{S}_{\mathrm{I}}\right]$ line are also predicted for metal-poor dwarf stars (Caffau \& Ludwig 2007).

Future investigations could test these possibilities from both the observational and theoretical side. For example, a thorough study analysing the forbidden line, the $\lambda 923 \mathrm{~nm}$ triplet, and the $\lambda 1045 \mathrm{~nm}$ triplet in an extended sample of cool metal-poor giants could firmly quantify the systematic differences between the diagnostics, assuming that they are real. Tailored non-LTE calculations using the most up-to-date model of the sulphur atom and the radiation field from MARCS models would test the differences of the non-LTE aspects of the lines more consistently. More calculations on the line formation of the three diagnostics in 3D stellar atmospheres would allow us to better quantify how reducing the problem to one dimension affects the lines.

Systematic effects concerning the different methods of deriving stellar parameters also deserve a closer inspection. Note that the $65 \mathrm{~K}$ difference between our $T_{\text {eff }}$ scale and the IQM scale makes the sulphur abundances derived in this work lower by about 0.02 dex than in the hypothetical case with no difference.

\subsubsection{Comparison with theoretical models}

In Fig. 6 we compare our results with the predictions of some of the Galactic chemical evolution models that have been published in the literature. In particular, we consider the models by Timmes et al. (1995), Kobayashi et al. (2006), Kobayashi et al. (2011), and Brusadin et al. (2013).

Timmes et al. (1995) considered a simple Galactic chemical evolution model where the Galaxy is formed by a collapse of a massive rotating gas cloud onto an exponential disk and a $1 / r^{2}$ bulge with a gas infall e-folding timescale of 4 Gyr. They assumed a Salpeter (1955) initial mass function, a quadratic Schmidt (1963) star formation rate, and instantaneous mixing of yields back into the interstellar medium. They adopted Type II supernovae $(\mathrm{SNe})$ yields from their companion paper (Woosley \& Weaver 1995), and yields from low-mass stars and Type Ia $\mathrm{SNe}$ from previous works by other authors. According to this model, at low metallicities sulphur is produced primarily in explosive oxygen-burning in Type II SNe.

Even though some of the assumptions can now be considered outdated, the model manages to reproduce the observed abundance trends for quite a few chemical elements between hydrogen and zinc when, as Timmes et al. (1995) themselves concluded, the iron yields calculated by Woosley \& Weaver (1995) are reduced by a factor of 2 . Of note is the fact that the model predicts a fairly shallow decline of $[\mathrm{S} / \mathrm{Fe}]$ starting from $[\mathrm{Fe} / \mathrm{H}] \sim-2$ (red lines in Figure 6) and a low enrichment of sulphur at all metallicities. The $[\mathrm{S} / \mathrm{Fe}]$ trend is relatively flat over the metallicity range between $-1<[\mathrm{Fe} / \mathrm{H}]<0$ because the additional sulphur produced in the more metal-rich Type II SNe (from progenitors with a total metal content above $10 \%$ solar) is balanced by the iron produced in Type Ia SNe.

Kobayashi et al. (2006) simulated the chemical evolution of the solar neighbourhood (using a one-zone model), Galactic bulge (infall model), thick disk (infall model), and the halo (closed-box model). They used their own nucleosynthesis yields 
for Type II supernovae and hypernovae, and the yields from their single-degenerate Type Ia supernovae models. Motivated by observational constraints (the observed $[\mathrm{O} / \mathrm{Fe}]$ versus $[\mathrm{Fe} / \mathrm{H}]$ ), they introduced a metallicity effect to delay the onset of SNe Ia in their simulations until $[\mathrm{Fe} / \mathrm{H}]=-1.1$.

Their simulations predict a slowly changing trend of $[\mathrm{S} / \mathrm{Fe}]$ below $[\mathrm{Fe} / \mathrm{H}] \simeq-1$, in the solar neighbourhood going from $[\mathrm{S} / \mathrm{Fe}] \simeq 0.5$ at $[\mathrm{Fe} / \mathrm{H}] \simeq-3$ to $[\mathrm{S} / \mathrm{Fe}] \simeq 0.37$ at $[\mathrm{Fe} / \mathrm{H}] \simeq-1$ caused by metal-poor and massive $\mathrm{SNe}$, which produce relatively larger amounts of sulphur. Above $[\mathrm{Fe} / \mathrm{H}] \simeq-1$ an almost linear decrease of $[\mathrm{S} / \mathrm{Fe}]$ is predicted (green solid line).

Kobayashi et al. (2011) used the same models as Kobayashi et al. (2006), but with some improvements, such as updated Type II SNe yields, and their chemical evolution model included yields from stellar winds and asymptotic giant branch stars (Karakas 2010). According to this and the Kobayashi et al. (2006) model, at low metallicities sulphur is chiefly produced in Type II SNe with a minor contribution from hypernovae.

Their results predict sulphur trends (Fig. 6, blue lines) that agree very well with their previous results for $[\mathrm{Fe} / \mathrm{H}]<-1$, while a higher $[\mathrm{S} / \mathrm{Fe}]$ is predicted at higher metallicities. The predicted differences between the solar neighbourhood (solid line), the thick disk (dashed line), and the Galactic halo (dotted line) are smaller than observed for other $\alpha$-elements, with the halo being at most 0.1 dex less enriched in sulphur than the thick disk at around $[\mathrm{Fe} / \mathrm{H}]=-0.5$.

Brusadin et al. (2013) adopted the two-infall model originally proposed by Chiappini et al. (1997), to which they added an outflow (wind) from halo stars. The two-infall model assumes that the Galaxy formed in two primary episodes of primordial gas accretion. During the first accretion episode the halo and the bulge are rapidly formed over a timescale of $\sim 1$ Gyr. In the second episode of much slower accretion the thin disk is formed from the inside out, that is, the accretion rate decreases with radius (the resulting timescale is $\sim 7 \mathrm{Gyr}$ at solar galactocentric radius). The outflow is added to reproduce the observed metallicity distribution function of the halo. Brusadin et al. (2013) adopted Type II SNe and hypernovae yields from Kobayashi et al. (2006) and Type Ia SNe yields from Iwamoto et al. (1999). In addition, they included the yields from asymptotic giant branch stars (Karakas 2010) and novae (Jose \& Hernanz 1998).

The main difference between the models with (Fig. 6, solid line) and without (dashed line) outflow is the knot at $[\mathrm{Fe} / \mathrm{H}] \simeq$ -1.3 predicted by the former, which is caused by a break in star formation soon after the first infall episode. This break coincides with the onset of the first Type Ia SNe $($ at $[\mathrm{Fe} / \mathrm{H}] \simeq-1.8)$ and, as a result, there is a rapid decline in the $[\alpha / \mathrm{Fe}]-[\mathrm{Fe} / \mathrm{H}]$ plane to lower $[\alpha / \mathrm{Fe}]$ values. Once the second infall episode starts, the overall metallicity decreases before star formation recommences and $[\alpha / \mathrm{Fe}]$ again increases, producing the knot.

When stars of all metallicities are considered, the results of this paper are best described by the predictions of the Kobayashi et al. (2011) models, which better describe the results for $[\mathrm{Fe} / \mathrm{H}]>-1$ than the Kobayashi et al. (2006) and Brusadin et al. (2013) models, which predict a similar sulphur enrichment at low metallicities. The Timmes et al. (1995) models are at odds with our results. However, the model with reduced iron yields (Fig. 6, red dashed line) fits the results of Nissen et al. (2007) very well. Of the two Brusadin et al. (2013) models, the one with no outflow fits our results slightly better at $[\mathrm{Fe} / \mathrm{H}]>-1$ and around the knot, but we only have a few stars with this metallicity.

In stars with $-1.5 \lesssim[\mathrm{Fe} / \mathrm{H}] \lesssim-1$ sulphur abundances from $[\mathrm{S} / \mathrm{Fe}] \sim 0$ to $[\mathrm{S} / \mathrm{Fe}] \sim 0.8$ have been determined in different studies in the literature. The explanation for finding low enrichment of sulphur might be the sampling of a low- $[\alpha / \mathrm{Fe}]$ halo population, evidence for which has been found both from observations of halo dwarfs (Nissen \& Schuster 2010) and theoretical $\Lambda \mathrm{CDM}$ simulations of the formation and evolution of the Galactic halo (Zolotov et al. 2010), according to which these low- $[\alpha / \mathrm{Fe}]$ stars have formed in satellite galaxies with lower star-formation rates and were subsequently accreted by the Milky Way. Distinct halo populations are not incorporated in the models discussed in this section.

The theoretical $[\mathrm{S} / \mathrm{Fe}]$ plateaus are slightly higher than we find in this study (except for the models of Timmes et al. 1995), but the agreement with theory is generally better than with the studies of Nissen et al. (2007) and Spite et al. (2011). The important point is that although detailed quantitative comparison between the models and observations seems premature because of the relatively large uncertainties in both, our results seem to support the notion that regular Type II SNe (with, perhaps, some contribution from hypernovae) can explain the Galactic chemical evolution of sulphur at low metallicities.

\section{Conclusions}

We have derived the sulphur abundances (or their upper limits) from spectrum synthesis of the [S I] $\lambda 1082.1 \mathrm{~nm}$ line in a sample of 39 stars spanning the stellar parameter ranges $3900 \lesssim T_{\text {eff }} \lesssim$ $5200 \mathrm{~K}, 0.5 \lesssim \log g \lesssim 3.0$ and $-2.4 \lesssim[\mathrm{Fe} / \mathrm{H}] \lesssim 0.2$ by employing one-dimensional local thermodynamic equilibrium MARCS model atmospheres.

The sulphur abundances show almost no correlation with metallicity for $[\mathrm{Fe} / \mathrm{H}] \lesssim-1$, which is typical for $\alpha$-elements. This means that the continued rise of $[\mathrm{S} / \mathrm{Fe}]$ below $[\mathrm{Fe} / \mathrm{H}] \simeq-1$ found in some previous studies is not supported by this study. This qualitatively agrees with contemporary models of Galactic chemical evolution. However, the uncertainties are large enough to warrant additional studies of the metallicity range $-1.5 \lesssim$ $[\mathrm{Fe} / \mathrm{H}] \lesssim-1$ with a much larger sample of stars.

We were able to derive homogeneous stellar parameters for 29 of the stars $(12$ with $[\mathrm{Fe} / \mathrm{H}]<-1)$ - we used photometric colour-effective temperature calibrations to derive $T_{\text {eff }}$, physical gravities with Bayesian estimation to derive $\log g$, and spectrum synthesis to derive $[\mathrm{Fe} / \mathrm{H}]$.

Our temperatures, derived from calibrations of González Hernández \& Bonifacio (2009), are systematically hotter than the average temperatures found in the literature by about $65 \mathrm{~K}$. At the same time, no systematic effects with respect to the literature were found for the surface gravities and metallicities derived here.

The forbidden sulphur line is a valuable diagnostic of sulphur abundances in giant and subgiant stars with $T_{\text {eff }} \lesssim 5200 \mathrm{~K}$ and $[\mathrm{Fe} / \mathrm{H}] \gtrsim-2.3$. The line is insensitive to the assumption of LTE and changes in $T_{\text {eff }}$, but is sensitive to changes in surface gravity and metallicity, which makes a homogeneous determination of the stellar parameters necessary. When results across multiple studies are compared, the differences in stellar parameters should be taken into account.

The uncertainties in the stellar parameters were found to dominate the total uncertainties in sulphur abundances. Therefore, the very high $\mathrm{S} / \mathrm{N}$ values of most of our spectra can be considered a luxury that in future observing campaigns might be sacrificed in favour of observing a larger sample of stars. An $S / N>100$ should be enough to derive precise sulphur abundances. 
The sulphur abundances derived in this work from the forbidden sulphur line at $\lambda 1082.1 \mathrm{~nm}$ are higher on average than the abundances derived from the triplet at $\lambda 923 \mathrm{~nm}$ in some previous studies (Nissen et al. 2007; Spite et al. 2011). This hints at systematic differences between the stellar parameter scales or the two sulphur diagnostics. In the latter case, we suspect these differences to be caused by non-LTE and 3D effects, but more detailed investigation is required.

A worthwhile undertaking would be obtaining a homogeneous set of stellar parameters for the stars in which very high (and low) sulphur abundances have been derived in the past, and re-determining the sulphur abundances in their atmospheres from the forbidden sulphur line when possible.

Acknowledgements. N.R. is a Royal Swedish Academy of Sciences Research Fellow supported by a grant from the Knut and Alice Wallenberg Foundation. Funds from Kungl. Fysiografiska Sällskapet i Lund and support from the Swedish Research Council, VR are acknowledged. The authors are grateful to L. Lindegren, S. Feltzing and the referee E. Caffau for providing valuable feedback that improved the quality of this paper.

\section{References}

Alonso, A., Arribas, S., \& Martínez-Roger, C. 1994, A\&AS, 107, 365 Alonso, A., Arribas, S., \& Martínez-Roger, C. 1998, A\&AS, 131, 209 Asplund, M., Grevesse, N., Sauval, A. J., \& Scott, P. 2009, ARA\&A, 47, 481 Bensby, T., Feltzing, S., Lundström, I., \& Ilyin, I. 2005, A\&A, 433, 185 Brusadin, G., Matteucci, F., \& Romano, D. 2013, A\&A, 554, A135 Caffau, E., \& Ludwig, H.-G. 2007, A\&A, 467, L11

Caffau, E., Bonifacio, P., Faraggiana, R., et al. 2005, A\&A, 441, 533

Caffau, E., Sbordone, L., Ludwig, H. G., Bonifacio, P., \& Spite, M. 2010, Astron. Nachr., 331, 725

Cardelli, J., Clayton, G., \& Mathis, J. 1989, ApJ, 345, 245

Cayrel, R., Depagne, E., Spite, M., et al. 2004, A\&A, 416, 1117

Chabrier, G. 2001, ApJ, 554, 1274

Chen, Y. Q., Nissen, P. E., Zhao, G., \& Asplund, M. 2002, A\&A, 390, 225

Chiappini, C., Matteucci, F., \& Gratton, R. 1997, ApJ, 477, 765

Collet, R., Asplund, M., \& Trampedach, R. 2007, A\&A, 469, 687

da Silva, L., Girardi, L., Pasquini, L., et al. 2006, A\&A, 458, 609

Drimmel, R., Cabrera-Lavers, A., \& López-Corredoira, M. 2003, A\&A, 409, 205

Francois, P. 1987, A\&A, 176, 294

Francois, P. 1988, A\&A, 195, 226

Froese Fischer, C., \& Tachiev, G. 2011, MCHF/MCDHF Database, Version 2, http://physics.nist.gov/mchf

Fuhrmann, K. 2008, MNRAS, 384, 173

Girardi, L., Bressan, A., Bertelli, G., \& Chiosi, C. 2000, A\&AS, 141, 371

González Hernández, J. I. \& Bonifacio, P. 2009, A\&A, 497, 497

Grevesse, N., Asplund, M., \& Sauval, A. J. 2007, Space Sci. Rev., 130, 105

Gustafsson, B., Edvardsson, B., Eriksson, K., et al. 2008, A\&A, 486, 951

Henyey, L., Vardya, M. S., \& Bodenheimer, P. 1965, ApJ, 142, 841
Hinkle, K., Wallace, L., \& Livingston, W. 1995, PASP, 107, 1042

Ilyin, I. V. 2000, Ph.D. Thesis, University of Oulu, Finland

Israelian, G., \& Rebolo, R. 2001, ApJ, 557, L43

Iwamoto, K., Brachwitz, F., Nomoto, K., et al. 1999, ApJS, 125, 439

Jørgensen, U. G., Larsson, M., Iwamae, A., \& Yu, B. 1996, A\&A, 315, 204

Jonsell, K., Edvardsson, B., Gustafsson, B., et al. 2005, A\&A, 440, 321

Jönsson, H., Ryde, N., Nissen, P. E., et al. 2011, A\&A, 530, A144

Jose, J., \& Hernanz, M. 1998, ApJ, 494, 680

Karakas, A. I. 2010, MNRAS, 403, 1413

Kobayashi, C., Umeda, H., Nomoto, K., Tominaga, N., \& Ohkubo, T. 2006, ApJ, 653,1145

Kobayashi, C., Karakas, A. I., \& Umeda, H. 2011, MNRAS, 414, 3231

Korn, A. J., \& Ryde, N. 2005, A\&A, 443, 1029

Korotin, S. A. 2009, Astron. Rep., 53, 651

Kupka, F. G., Ryabchikova, T. A., Piskunov, N. E., Stempels, H. C., \& Weiss, W. W. 2000, Balt. Astron., 9, 590

Lind, K., Bergemann, M., \& Asplund, M. 2012, MNRAS, 427, 50

Mashonkina, L., Gehren, T., Shi, J.-R., Korn, A. J., \& Grupp, F. 2011, A\&A, 528, A87

McCall, M. L. 2004, AJ, 128, 2144

McWilliam, A. 1997, ARA\&A, 35, 503

Mermilliod, J.-C., Mermilliod, M., \& Hauck, B. 1997, A\&AS, 124, 349

Nakamura, T., Umeda, H., Iwamoto, K., et al. 2001, ApJ, 555, 880

Nissen, P. E., \& Schuster, W. J. 2010, A\&A, 511, L10

Nissen, P. E., Chen, Y. Q., Asplund, M., \& Pettini, M. 2004, A\&A, 415, 993

Nissen, P. E., Akerman, C., Asplund, M., et al. 2007, A\&A, 469, 319

Querci, F., Querci, M., \& Kunde, V. G. 1971, A\&A, 15, 256

Ramaty, R., Scully, S. T., Lingenfelter, R. E., \& Kozlovsky, B. 2000, ApJ, 534, 747

Ramírez, I., \& Allende Prieto, C. 2011, ApJ, 743, 135

Ramírez, I., \& Meléndez, J. 2005a, ApJ, 626, 446

Ramírez, I., \& Meléndez, J. 2005b, ApJ, 626, 465

Rossi, S., Beers, T. C., Sneden, C., et al. 2005, AJ, 130, 2804

Ryde, N. 2006, A\&A, 455, L13

Ryde, N., \& Lambert, D. L. 2004, A\&A, 415, 559

Salpeter, E. E. 1955, ApJ, 121, 161

Schmidt, M. 1963, ApJ, 137, 758

Skrutskie, M. F., Cutri, R. M., Stiening, R., et al. 2006, AJ, 131, 1163

Soubiran, C., Le Campion, J.-F., Cayrel de Strobel, G., \& Caillo, A. 2010, A\&A, 515, A 111

Spite, M., Caffau, E., Andrievsky, S. M., et al. 2011, A\&A, 528, A9

Takada-Hidai, M., Takeda, Y., Sato, S., et al. 2002, ApJ, 573, 614

Takada-Hidai, M., Saito, Y.-J., Takeda, Y., et al. 2005, PASJ, 57, 347

Takeda, Y., Hashimoto, O., Taguchi, H., et al. 2005, PASJ, 57, 751

Taylor, B. J. 1986, ApJS, 60, 577

Timmes, F. X., Woosley, S. E., \& Weaver, T. A. 1995, ApJS, 98, 617

Valenti, J. A., \& Piskunov, N. 1996, A\&AS, 118, 595

van Leeuwen, F. 2007, A\&A, 474, 653

Wallace, L., Hinkle, K., \& Livingston, W. C. 1993, An atlas of the photospheric spectrum from 8900 to $13600 \mathrm{~cm}^{-1}$ (7350 to $11230 \AA$ A), NSO Technical Report \# 93-001

Wallerstein, G., \& Conti, P. 1964, ApJ, 140, 858

Woosley, S. E., \& Weaver, T. A. 1995, ApJS, 101, 181

Zolotov, A., Willman, B., Brooks, A. M., et al. 2010, ApJ, 721, 738 
A\&A 559, A115 (2013)

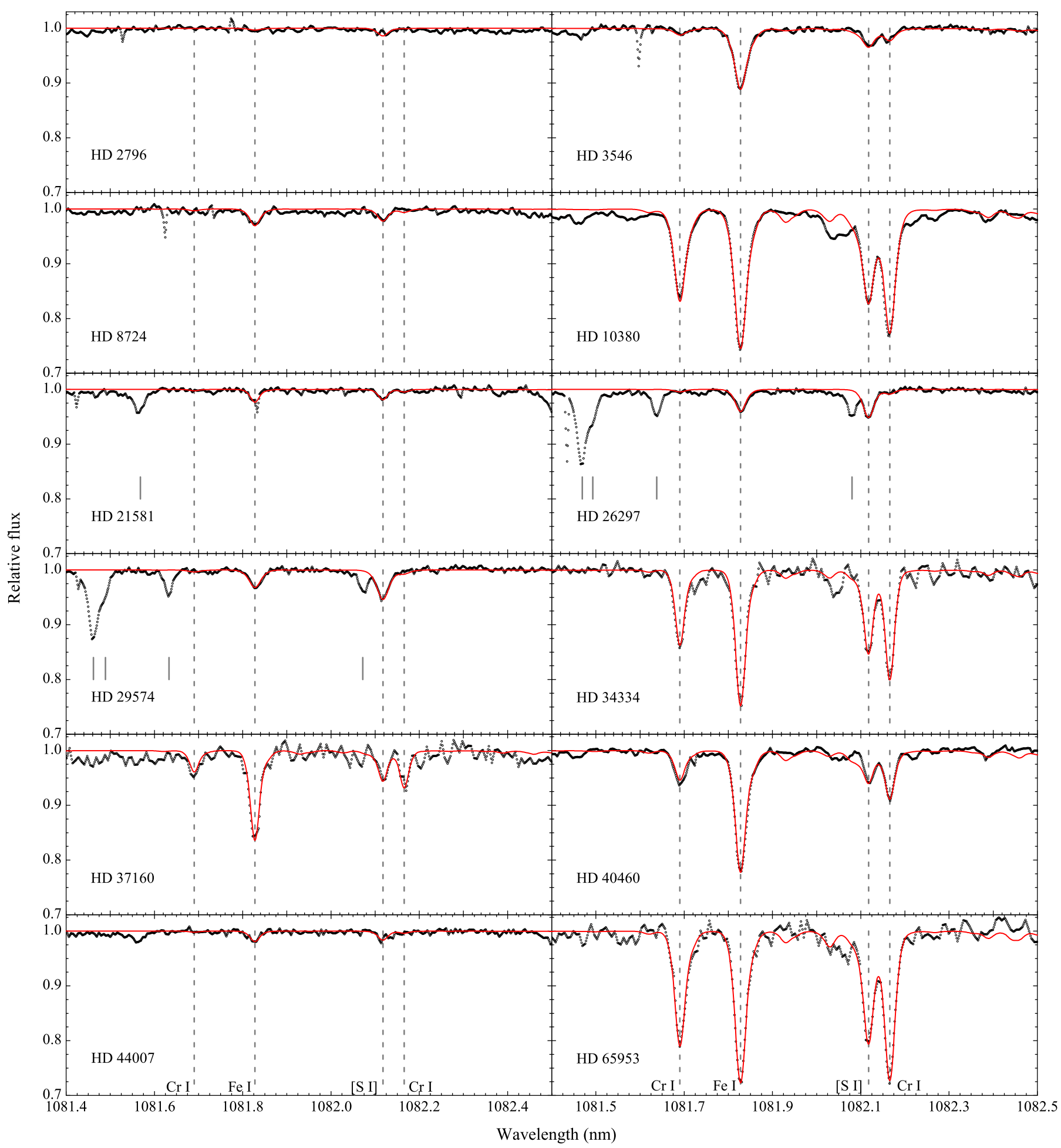

Fig. 3. Synthetic spectra (red line) plotted over the observations (black circles) for a sample of the stars. The positions of important lines are marked by vertical dashed lines. The vertical ticks mark the positions of telluric lines. 
E. Matrozis et al.: Galactic chemical evolution of sulphur

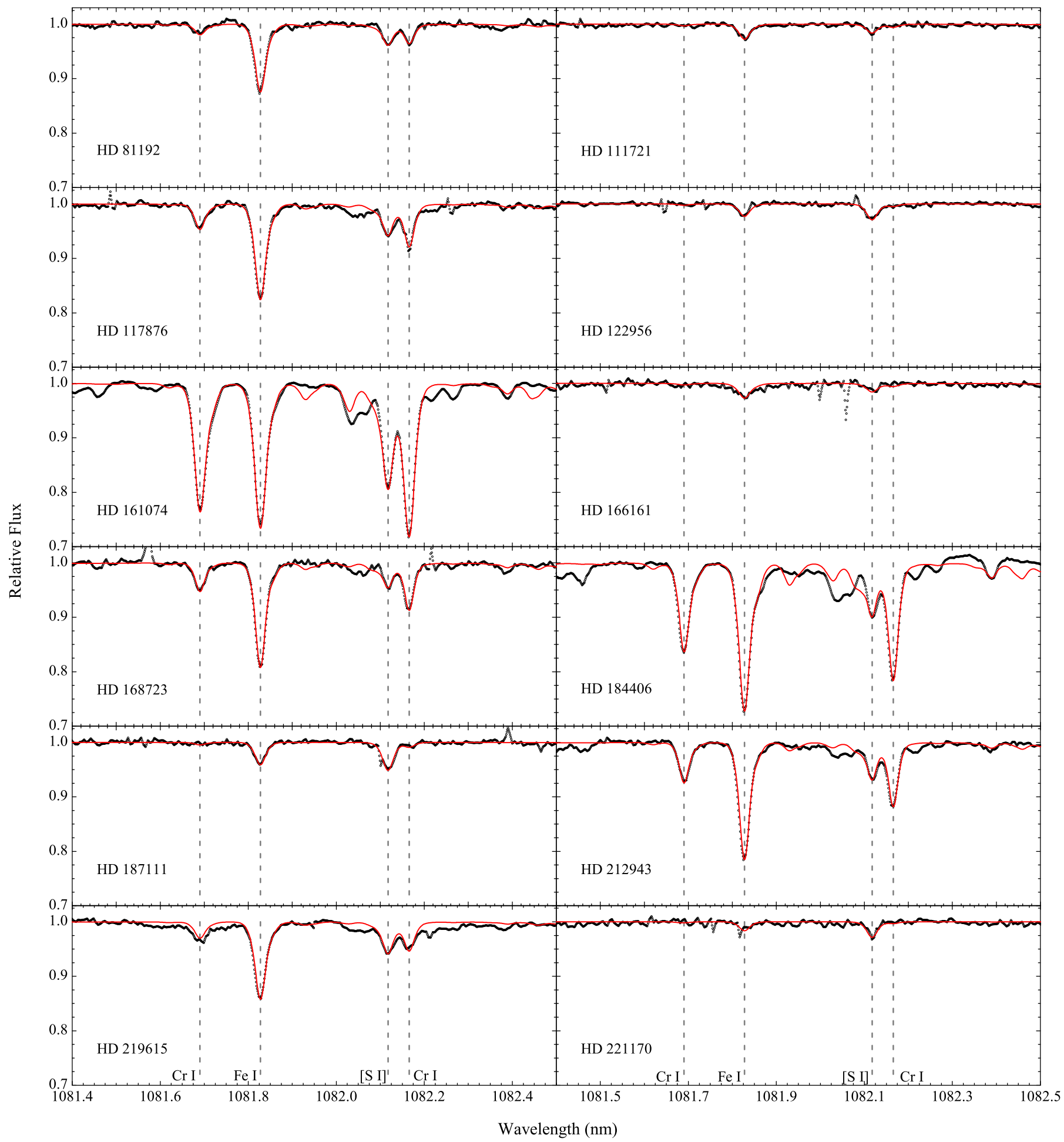

Fig. 4. Synthetic spectra (red line) plotted over the observations (black circles) for a sample of the stars. The positions of important lines are marked by vertical dashed lines. 